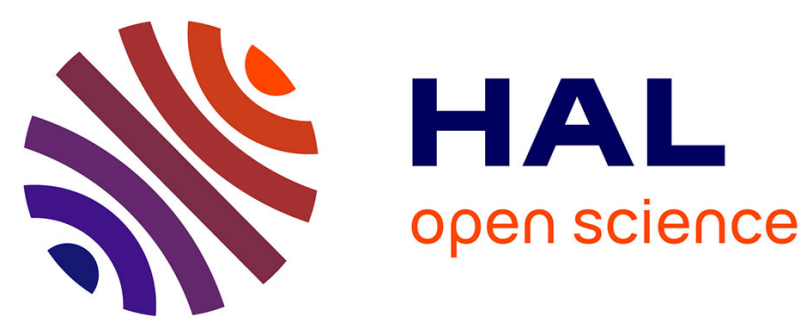

\title{
Offline parameter estimation using EnKF and maximum likelihood error covariance estimates: Application to a subgrid-scale orography parameterization
}

Pierre Tandeo, Manuel Pulido, François Lott

\section{- To cite this version:}

Pierre Tandeo, Manuel Pulido, François Lott. Offline parameter estimation using EnKF and maximum likelihood error covariance estimates: Application to a subgrid-scale orography parameterization. Quarterly Journal of the Royal Meteorological Society, 2015, 141 (687), pp.383 - 395. 10.1002/qj.2357 . hal-01194557

\section{HAL Id: hal-01194557 \\ https://hal.science/hal-01194557}

Submitted on 7 Sep 2015

HAL is a multi-disciplinary open access archive for the deposit and dissemination of scientific research documents, whether they are published or not. The documents may come from teaching and research institutions in France or abroad, or from public or private research centers.
L'archive ouverte pluridisciplinaire HAL, est destinée au dépôt et à la diffusion de documents scientifiques de niveau recherche, publiés ou non, émanant des établissements d'enseignement et de recherche français ou étrangers, des laboratoires publics ou privés. 


\title{
Offline parameter estimation using EnKF and maximum likelihood error covariance estimates: Application to a subgrid-scale orography parameterization
}

\author{
Pierre Tandeo ${ }^{1,2}$, Manuel Pulido ${ }^{2}$ and François Lott ${ }^{3}$ \\ ${ }^{1}$ CNRS UMR 6285 LabSTICC, pôle CID, Telecom Bretagne, Brest, France, \\ pierre.tandeo@telecom-bretagne.eu \\ ${ }^{2}$ Department of Physics, Universidad Nacional del Nordeste, Corrientes, Argentina \\ ${ }^{3}$ Laboratoire de Meteorologie Dynamique, Ecole Normale Superieure, Paris, France
}

April 9, 2015

keywords Offline parameter estimation; EnKF; EM algorithm; Subgrid-scale orography parameterization

\begin{abstract}
Recent works show that the parameters controlling the parameterizations of the physical processes in climate models can be estimated from observations using filtering techniques. In this paper, we propose an offline parameter estimation approach, without estimating the state of the climate model. It is based on the Ensemble Kalman Filter (EnKF) and an iterative estimation of the error covariance matrices and of the background state using a maximum likelihood algorithm. The technique is implemented in a subgridscale orography (SSO) parameterization scheme that works in a single vertical column. First, the parameter estimation technique is evaluated using twin experiments. Then, the technique is used with synthetic observations to estimate how the parameters of the SSO scheme should change when the resolution of the input orography dataset of a general circulation model is increased. Our analysis reveals that when the resolution of the orography dataset increases, the
\end{abstract}

scheme should take into account the dynamical sheltering that can occur at low levels between mountain peaks located within the same gridbox area.

\section{Introduction}

Numerical models including atmospheric/oceanic General Circulation Models (GCMs) and current earth system models, contain several physical parameterizations with a large number of parameters. Climate predictions using these numerical models are sensitive to the large set of parameters that are present in the physical parameterizations (cf. [Stainforth et al.(2005)]). Most of these unknown physical parameters can not be determined directly from observations and are generally manually tuned. This subjective approach is excessively time demanding and do not give optimal results. Moreover, if the horizontal resolution of the model or of an input dataset is increased or a parameterization scheme is changed, the physical parameters need to be re-evaluated. To address these issues, several authors (see e.g. [Jackson et al.(2004)] and [Severijns and Hazeleger(2005)]) propose to estimate the physical parameters objectively, defining 
a cost function based on the Root Mean Square Error (RMSE) criterion. The idea is to find the optimal set of parameter values that gives the minimum RMSE and produces the lowest model error. However, nonlinear model responses may produce multiple local minima in the cost function (cf. [Posselt and Bishop(2012)]), and thus sophisticated optimization algorithms are required to find the global minimum corresponding to the optimal parameters. Such optimization algorithms are usually too expensive computationally to be employed in sophisticated models. An alternative consists in supposing that the parameters are stochastic and in estimating them using filtering techniques (see e.g. [Annan and Hargreaves(2004)], [Posselt and Bishop(2012)], [Ruiz et al.(2013)] and [Schirber et al.(2013)]). The basic idea to estimate the parameters is based on an augmented state composed by both the state of the system and the physical parameters in a nonlinear Gaussian state-space model. This online estimation is a tough problem in practice. Even a simple linear state equation with multiplicative parameters behaves nonlinearly for parameter estimation ([Yang and Delsole(2009)]).

Another approach consists in estimating the physical parameters independently of the state of the system. The particular advantage of using an offline estimation technique is that the control space is reduced from $10^{7}$ to just a few dimensions. This drastic reduction in size permits to conduct several model/parameterization evaluations as is often needed in parameter estimation. One disadvantage of offline techniques, is that they can not take into account the feedback of the changes that the parameterization produces onto the parameterization itself. For a subgrid-scale orography (SSO) scheme nevertheless, this issue should not be too critical, since most of the flow changes produced are advected downstream (for instance in the form of potential vorticity banners, see Figure 13(c) in $[\operatorname{Lott}(1995)])$. Accordingly, the feedback can be neglected if the mountains considered are not close to the lee of other mountains.

In order to conduct an offline estimation of physical parameters, the parameterization should be compared to observations, for instance the PYREX campaign, in which surface drag and momentum fluxes were measured over a transect of the Pyrénées mountain (cf. [Bougeault et al.(1990)]). In this case, the mountain massif can be considered to be entirely located within a model gridbox area of a climate model, so drag and momentum flux can be directly compared to the same quantities predicted by the scheme over the same area. Therefore, we can validate SSO schemes using a single vertical column. This approach is often used prior to the implementation of the schemes in GCMs (see for instance the offline tests of the scheme in single vertical columns using the PYREX data in [Lott and Miller(1997)]).

[Pulido and Thuburn(2005)] and [Pulido and Thuburn(2008)] showed that a fourdimensional variational data assimilation technique can be used to estimate the missing momentum forcing due to the unresolved/subgrid-scale gravity waves in the stratosphere. This missing momentum forcing was used to estimate optimal parameters of a nonorographic gravity wave parameterization in [Pulido et al.(2012)]. Using twin experiments, they showed that the variational data assimilation technique does not converge towards the optimal parameters because of the nonlinear response of the parameterization to parameter perturbations. They employed a time-demanding genetic algorithm to overcome these difficulties. In the present work, we propose a similar offline parameter estimation procedure but using a ensemble-based data assimilation technique to estimate the optimal parameters of a SSO scheme.

The technique presented here uses the Ensemble Kalman Filter (EnKF) and Ensemble Kalman Smoother (EnKS) which are reviewed in detail in [Evensen(2009)]. In this work, we do not use an augmented state to estimate parameters of GCMs as is usually done for online estimation like in [Annan and Hargreaves(2007)] or [Ruiz et al.(2013)], instead the state variables for the EnKF are only the physical parameters in this offline parameter estimation. As we do not have any knowledge of their temporal evolution, the state model is supposed to follow a random walk. In this way, we assume a non-negligible model error. An innovative part of our technique is that we also estimate the statistical parameters of the EnKF: (i) the covariance matrices 
of the Gaussian errors that control the weight of the state and the observation equations and (ii) the background state of the filter, typically an a priori knowledge of the physical parameters. Generally, these statistical parameters of the EnKF are prescribed values chosen by the user. In practice, this manual tuning does not ensure the filter convergence to the state of the system. To overcome this problem, the standard implementations of the EnKF use an inflation factor for the forecast and/or observational error covariance matrices to avoid filter divergence. However, the main problem of this approach is the choice of the covariance inflation (additive or multiplicative) and the amplitude of the inflation. Several studies propose to estimate the inflation factors using the first moment estimation of the squared innovation (see e.g. [Wang and $\operatorname{Bishop}(2003)]$, [Li et al.(2009)] and [Liang et al.(2011)]), Bayesian approaches (see e.g. [Anderson(2007)] and [Miyoshi(2011)]) or the second-order least squares statistic of the squared innovation as in [Wu et al.(2012)]. The technique presented in this paper does not need to use any inflation factor since the statistical parameters are nondeterministic values. Here, as the estimation is offline in a low-dimensional system, we estimate directly the entire error covariance matrices and the background state of the EnKF using a maximum likelihood approach. In particular, we use the iterative and efficient Expectation-Maximization (EM) algorithm introduced by [Dempster et al.(1977)]. To our knowledge, the implemented technique in this work based on the combination of an ensemble Kalman filter with the Expectation-Maximization algorithm has not been proposed previously in data assimilation.

The novel estimation technique is applied to the SSO scheme described in [Lott and Miller(1997)] and revised in $[\operatorname{Lott}(1999)]$. This SSO scheme computes the wind tendencies due to the subgrid-scale orography and is implemented in three GCMs: the Laboratoire de Météorologie Dynamique (LMDz), the ECHAM model which is the atmospheric component of the Earth System Model of the MaxPlanck Institute (MPI-ESM) and the European Centre for Medium-Range Weather Forecasts (ECMWF) model. It is known that weather forecast and cli- mate models are sensitive to the physical parameters of SSO schemes (see e.g. [Palmer et al.(1986)], [Lott et al.(2005)] and [Sigmond et al.(2008)] respectively). Currently, this issue is still important since climate models now extent to the middle atmosphere where mountain gravity waves significantly affect the Brewer-Dobson circulation ([McLandress and Shepherd(2009)]). This circulation seems to intensify with the climate change ([Li et al.(2008)]). These results call for a reevaluation of the SSO schemes in the middleatmosphere resolving models and in particular of the set of parameters used in the schemes. An optimization of the SSO schemes can help to evaluate better the potential effects of the orographic gravity wave drag on the westerlies in mid-latitudes.

This paper is organized as follows. First, we describe the SSO scheme and the data-sets it uses in Section 2. Then, in Section 3, we present the statistical model used to estimate the physical parameters of the SSO scheme. The details of the estimation technique based on the EnKF, EnKS and the EM algorithm are explained in Section 4. The estimation technique is applied to a column version (not a 3D version) of the subgrid-scale orography scheme. We then use two synthetic cases (i.e., without using real observations): an identical twin-experiment and a situation in which the horizontal resolution of the orography dataset is changed. We show the results in Section 5. Conclusions are drawn and perspective work is outlined in Section 6. In general, the unified notations of data assimilation given in [Ide et al.(1997)] are used in this paper.

\section{$2 \quad$ Data and model}

\subsection{General circulation model data}

To conduct our offline estimation we used daily data from a simulation done with the LMDz GCM ([Hourdin et al.(2006)]) using a horizontal resolution of $3.75^{\circ} \times 2.5^{\circ}$ and 50 vertical levels with a model top at $5 \mathrm{hPa}$. We have extracted from this model the SSO scheme we want to optimize. To conduct the optimization, we limit ourselves to a one month 
period, July 2000. The exact year itself is of little importance, since the run considered has a spin up of several years, and was not constrained by other forcings than the sea-surface temperature and the landsea ice cover. The particular month chosen is in mid winter, when high wind speed conditions prevail over the Southern Andes.

The SSO scheme we use represents mountain gravity wave drag and blocked flow drag following [Lott and Miller(1997)]. It also introduce lateral lift to take into account the fact that narrow valleys are partially sheltered from the large scale winds in the free troposphere $(\mathrm{cf}$. $[\operatorname{Lott}(1999)])$. The scheme was extended to the stratosphere in [Lott et al.(2005)] and this is the version that we use. For completeness, the salient features of scheme are described here.

Before launching a simulation, ten subgrid scale orography parameters are calculated in each model gridbox: the mountain minimum, mean, and maximum elevations, the mountain departure from the mean is then characterized by its anisotropy, its orientation angle, its slope and its standard deviation. As we will see, when we change orography datasets these parameters change significantly and the most dramatic changes concern the evaluation of the slope. We will adress these issues in Sect. 5.2 and evaluate the changes to be done to the SSO scheme used in $\mathrm{LMDz}$, when we make a transition from the $10 \mathrm{~min}-$ utes of resolution US Navy orography dataset used in most current applications, to a more refined 2 minutes of resolution dataset. At each time step, the SSO scheme uses the background flow conditions predicted by the model at a given gridpoint (i.e. the horizontal components of the winds, the temperature and the surface pressure), and predicts the effect of the SSO on the large-scale flow at all model levels.

The SSO scheme uses a set of six non-dimensional parameters of order $\mathrm{O}(1)$ which characterize the mesoscale and synoptic-scale effects of the mountain on the large-scale flow. The first three parameters $G$, $C_{d}$ and $C_{l}$ directly scale the forces associated to the different processes parameterized: the gravity wave drag, the low-level blocked flow drag, and the lowlevel lift that enhances large-scale vortex compression to represent valleys sheltering respectively. The other three parameters $H_{N C}, \beta$ and $R i_{c}$, are used
Table 1: Physical parameters of the SSO scheme, their assigned true values and their corresponding physical range.

\begin{tabular}{ccc}
\hline Physical parameters & True & Range \\
\hline$G$ & 1 & $(0,1.5)$ \\
$C_{d}$ & 1 & $(0,1.5)$ \\
$C_{l}$ & 1 & $(0,1.5)$ \\
$H_{n c}$ & 1 & $(0,1.5)$ \\
$\beta$ & 0.5 & $(0,1)$ \\
$R i_{c}$ & 0.25 & $(0,2)$ \\
\hline
\end{tabular}

to characterize the low-level flow blocking depth, the fraction of the gravity wave drag that propagates toward the free troposphere and aloft, and the critical Richardson number that is used to predict when the mountain waves break. In Table 1, we give the values of each parameter used in operation, and also the range of values we will consider as plausible when we will re-tune the SSO scheme.

The profiles of wind tendencies given by the SSO scheme are very sensitive to the value of the six nondimensional parameters as in other schemes. The set of parameters used in the past were motivated by decades of research on mountain flow dynamics, and by few experimental campaigns conducted over specific areas like the Pyrenees in France (for a motivation of the lift based on the PYREX campaign, see for instance $[\operatorname{Lott}(1995)])$. Although satellite data combined with high-resolution simulations could also be used in the future ([Hertzog et al.(2012)]), it remains that local tunings will probably still be needed, at least near places where the drag forces can potentially be very important. For this reason, and also because the methodology we propose is well adapted to handle 1-column models ([Posselt and Bishop(2012)]), this is the strategy we have followed in the present paper, where we imagine that an observational campaign takes place near the Perito Moreno Glacier in the Andes ( $46^{\circ}$ south, $71^{\circ}$ west), location represented by a dot in Figure 1. There, the mountains are characterized by an important anisotropic shape and strong variations of the altitudes (the standard deviation is $295 \mathrm{~m}$ for a mean altitude of $531 \mathrm{~m}$ and a peak of $1513 \mathrm{~m}$ ). These topographical conditions represented 
in Figure 1(a) are ideal to study mountain induced forces especially in high surface wind speed conditions such as in Figure 1(b). Indeed, this geographical location gave one of the largest subgrid-scale mountain drag amplitudes on the Earth in a preliminary spatial analysis for July 2000 in which we computed globally the subgrid-scale mountain drag with the scheme.

\subsection{Preliminary tests}

To give a preliminary view of the scheme outputs, Figure 2 shows the tendencies predicted by the SSO scheme,

$$
\mathbf{y}\left(t_{k}\right)=\mathcal{F}\left(\boldsymbol{\theta}, \mathbf{Z}\left(t_{k}\right)\right)
$$

where $\boldsymbol{\theta}=\left(H_{N C}, C_{d}, R i_{c}, G, C_{l}, \beta\right)$, and $\mathbf{Z}\left(t_{k}\right)$ is a generic notation for the vertical profiles of the horizontal winds and temperature. In (1), the vector $\mathbf{y}\left(t_{k}\right)$ has $m=100$ values each of the 31 days $k \in\{1, \ldots, K=31\}$ of July 2000. Each day, the first 50 values correspond to the zonal tendencies at the 50 model levels, and the last 50 values to the meridional tendencies.

From a preliminary temporal analysis during July 2000 on the chosen location, we distinguish two characteristic regimes of wind profiles in terms of the resulting induced SSO tendencies. Two examples of these regimes are shown in Figure 3. On the 5 July 2000 (dotted line), the wind profile shows low and constant wind speeds with the altitude. On the 25 July (dashed line) the profile shows higher surface wind speeds and an increase of the wind with height, due to the presence of the subpolar jet in the region. The zonal and meridional components of the SSO tendency for the two cases are shown in Figure 2. The free physical parameters of the scheme are set to $H_{N C}^{t}=1, C_{d}^{t}=1, R i_{c}^{t}=0.25, G^{t}=1, C_{l}^{t}=1$ and $\beta^{t}=0.5$. These set of "true" parameter values $\boldsymbol{\theta}^{t}$ were proposed by $[\operatorname{Lott}(1999)]$ and generate the "true" tendency denoted $\mathbf{y}^{t}\left(t_{k}\right)$. From Figure 2, on the 25 July 2000, we find large tendencies whereas on the 5 July 2000 the effect is much weaker due to the low wind speed conditions. The $95^{\text {th }}$ percentile envelope around the mean value for the month of July 2000 is also shown. It indicates that the pre-

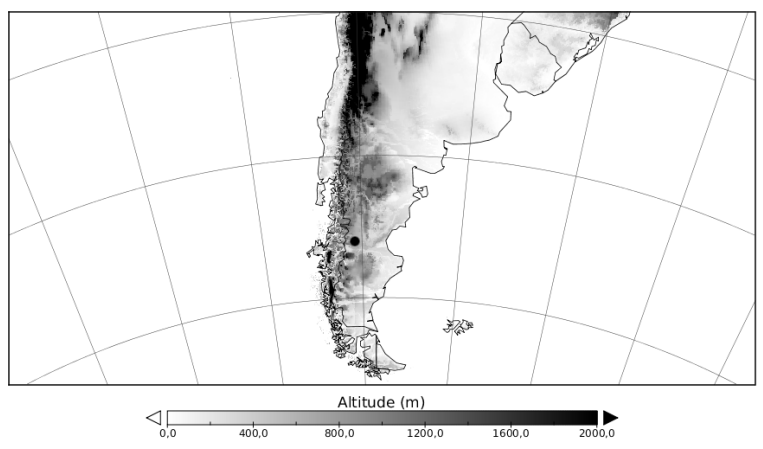

(a)

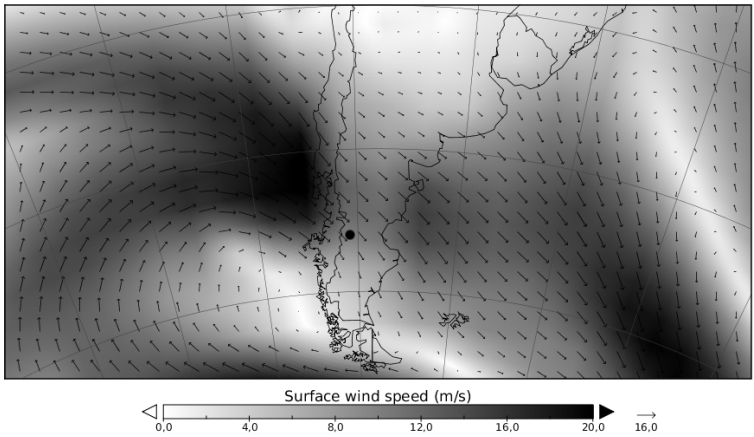

(b)

Figure 1: (a) Topography of the south Andes and (b) surface winds the 25 July 2000. The location of the chosen mountain peaks is close to the Perito Moreno Glacier ( $46^{\circ}$ south, $71^{\circ}$ west) and represented by a dot. 
dicted SSO tendencies tend to be small in the midlevels and larger at levels corresponding to the peak of the mountain $(800 \mathrm{hPa})$ and to the tropospheric jet $(250 \mathrm{hPa})$.

To evaluate how the outputs of the scheme vary with the different parameters, we evaluate the cost function

$$
J\left(t_{k}\right)=\left(\mathbf{y}^{t}\left(t_{k}\right)-\mathbf{y}\left(t_{k}\right)\right)^{\top}\left(\mathbf{y}^{t}\left(t_{k}\right)-\mathbf{y}\left(t_{k}\right)\right)
$$

where the transpose notation ${ }^{\top}$ is used, so that the square differences are summed over all altitudes and over the two components (zonal and meridional). Firstly, the cost function given in (2) is computed by changing one physical parameter and by fixing the other parameters to the true values.

Figure 4(a) shows the sensitivity of $J$ as a function of $H_{N C}$ and $C_{l}$ for the state found on 5 July 2000 which is a situation with low surface winds as shown in Figure 3. The cost function associated with $H_{N C}$ parameter shows a nonquadratic behavior, representing a nonlinear sensitivity in the derivative of $J$. The parameter $C_{l}$ shows a quadratic cost function so that its sensitivity is linear. The other four parameters also show a linear sensitivity, as found for the $C_{l}$ parameter, so that their cost functions are not shown. Figure 4(b) shows the sensitivity of $J$ in high surface wind speed conditions, on the 25 July 2000. The sensitivity of $J$ for $H_{N C}$ parameter is enhanced by a factor of $10^{3}$ in strong wind speed conditions compared to the sensitivity of the weak wind speed condition case. Finally, a relatively weaker enhancement of the $J$ sensitivity is found for high surface wind speed conditions to certain physical parameters, e.g. $C_{l}$ (squares) and $C_{d}$ (not shown here) compared to the enhancement of $H_{N C}$ sensitivity between low and high surface wind conditions. In Figure 4 (b), a saturation of the cost function is found close to the global minimum for $H_{N C}>1.1$ (circles) in high wind speed conditions. This behaviour can be explained as follows. As the surface wind increases and $H_{N C}$ increases the blocked flow depth decreases and eventually reaches 0 (see (4) and (9) in [Lott and Miller(1997)]). At this point, the parameter $H_{N C}$ becomes saturated since an increase of its value can not change the blocked flow depth to neg-

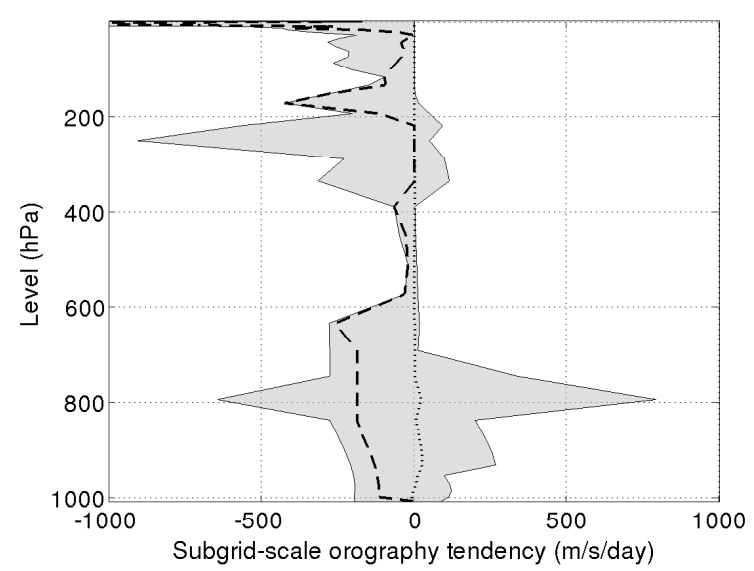

(a)

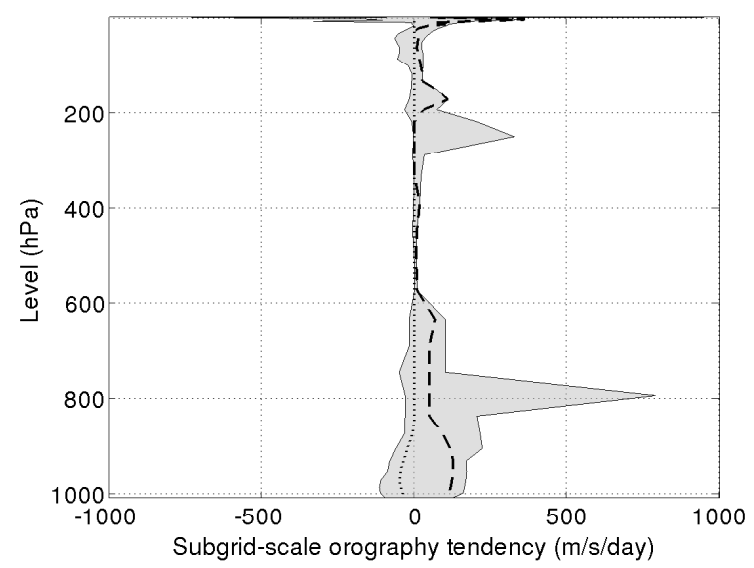

(b)

Figure 2: Vertical profiles of (a) zonal and (b) meridional tendencies generated by the SSO scheme at location $46^{\circ}$ south, $71^{\circ}$ west within the Andes. The gray intervals correspond to the $95^{t h}$ percentile envelope on the month of July 2000. The dotted and dashed lines correspond respectively to the mountain drag profiles the 5 and 25 July 2000. 


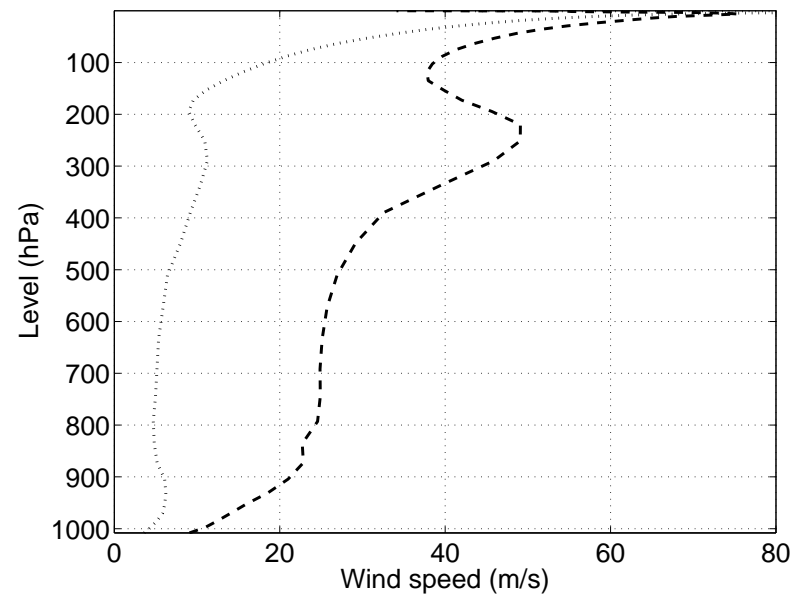

Figure 3: Wind speed profiles from the LMDz GCM model the 5 (dotted line) and 25 (dashed line) July 2000 at location $46^{\circ}$ south, $71^{\circ}$ west within the Andes.

ative values. Therefore, $H_{N C}$ values larger than this critical value cannot affect the SSO predictions.

In a second sensitivity experiment, the cost function given in (2) is computed changing two physical parameters simultaneously. Figure 5 shows the cost function as a function of the physical parameters $H_{N C}$ and $G$. Parameter $H_{N C}$ is correlated with $G$ parameter. The 10 smallest values of the cost function are indicated in the Figure 5 with black dots. They underline the fact that the global minimum region of the cost function (intersection of the 2 dashed black lines) is not well defined. On the contrary, in Figure 5(a), a large region of very low sensitivity close to the global minimum is highlighted, especially in low wind speed conditions where the sensitivity of $J$ is reduced. In this region of the cost function, there is a negative correlation between $H_{N C}$ and $G$.

\section{Nonlinear Gaussian state- space model}

To estimate the $n=6$ physical parameters in $\boldsymbol{\theta}$ via our filtering technique, we first need to make them stochastic. We denote them as $\mathbf{x}$ and we say it is the

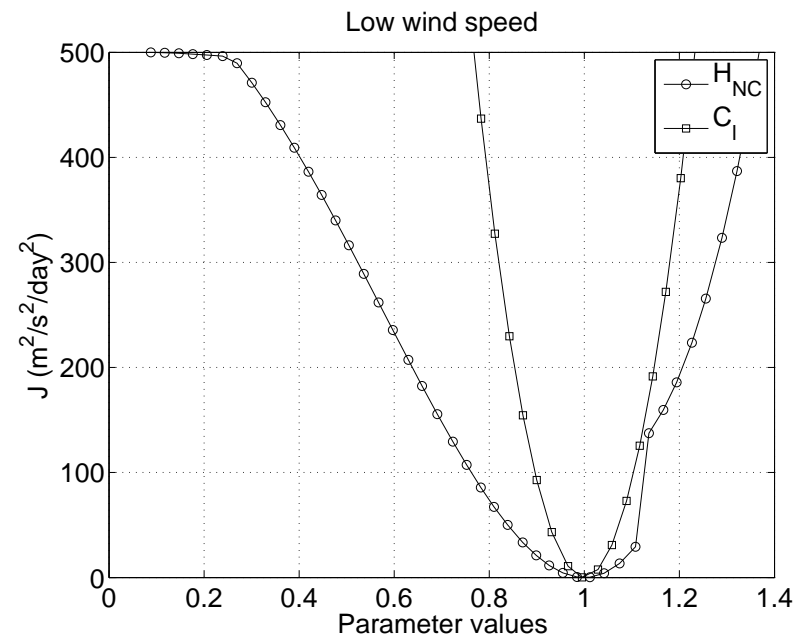

(a)

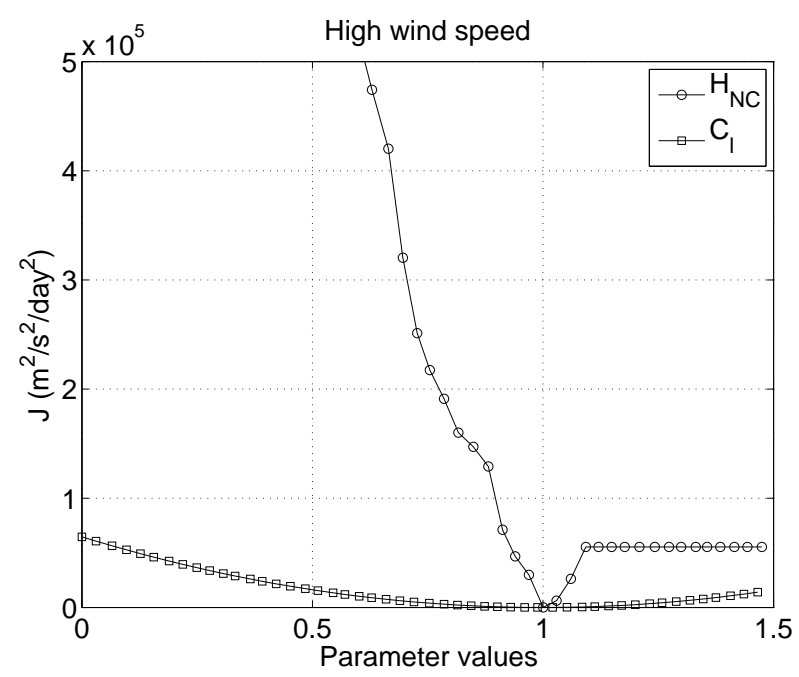

(b)

Figure 4: Cost function (2) as a function of the physical parameters $H_{N C}$ (circles) and $C_{l}$ (squares). The true values of the physical parameters are $H_{N C}^{t}=1$ and $C_{l}^{t}=1$. The results are given for the location $46^{\circ}$ south, $71^{\circ}$ west, (a) on 5 July 2000 representing low surface wind speed conditions and (b) on 25 July 2000 representing high surface wind speed conditions. 


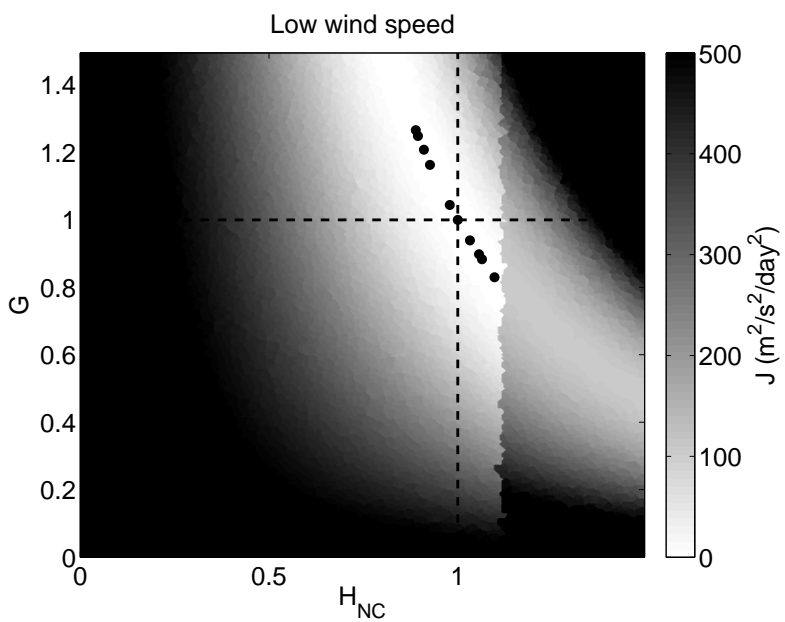

(a)

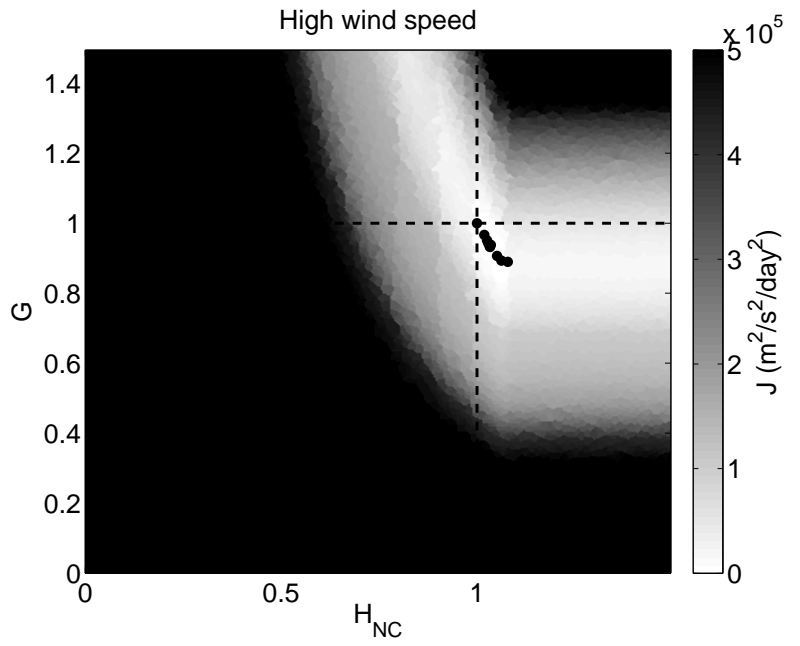

(b)

Figure 5: Cost function (2) as a function of the physical parameters $H_{N C}$ (x-axis) and $G$ (y-axis). The intersection of dashed black lines corresponds to the true physical parameters $H_{N C}^{t}=1$ and $G^{t}=1$. The black dots correspond to the physical parameters that generate the 10 minima of the cost function. The results are given for the location $46^{\circ}$ south, $71^{\circ}$ west, (a) on 5 July 2000 representing low surface wind speed conditions and (b) on 25 July 2000 representing high surface wind speed conditions. "state of the system". The state evolution is given by a Gaussian random walk,

$$
\mathbf{x}\left(t_{k}\right)=\mathbf{x}\left(t_{k-1}\right)+\boldsymbol{\eta}\left(t_{k}\right),
$$

where the $n$-dimensional stochastic random vector $\left\{\boldsymbol{\eta}\left(t_{k}\right)\right\}_{k \in\{1, \ldots, K\}}$ represents an additive perturbation at each time $t_{k}$. We assume that the perturbations are Gaussianly distributed with zero mean and a constant in time $n \times n$ covariance matrix $\mathbf{Q}$. Equation (3) is taken as the state equation in our state-space model.

If we use directly the physical parameters $\boldsymbol{\theta}$ as the state of the system, they can easily become negative, or reach very large values whereas the parameters in the SSO scheme are assumed to be always positive and of the order of unity. For this reason, we map the physical parameters $\boldsymbol{\theta}$ on $\mathbf{x}$ by using the Gauss error function $\boldsymbol{\theta}=\mathcal{G}(\mathbf{x})$, as sometimes used in data assimilation ([Hu et al.(2010)]).

At the initial time of (3), we introduce an a priori knowledge of the physical parameters. We assume that this background information follows a Gaussian distribution given by the $n$-dimensional vector mean $\mathbf{x}^{b}$ and the $n \times n$ covariance matrix $\mathbf{B}$.

At time $t_{k}$, the zonal and meridional SSO tendencies given in (1) are assumed to be observed. They are stored in the $m$-dimensional stochastic random vector $\left\{\mathbf{y}\left(t_{k}\right)\right\}_{k \in\{1, \ldots, K\}}$. The state vector at time $t_{k}$ is related to the observation by means of the observation equation defined by

$$
\mathbf{y}\left(t_{k}\right)=\mathcal{H}_{k}\left(\mathbf{x}\left(t_{k}\right)\right)+\boldsymbol{\epsilon}\left(t_{k}\right),
$$

where the observation operator $\mathcal{H}_{k}$ is the nonlinear function defined by

$$
\mathcal{H}_{k}\left(\mathbf{x}\left(t_{k}\right)\right)=\mathcal{F}\left(\mathcal{G}\left(\mathbf{x}\left(t_{k}\right)\right), \mathbf{Z}\left(t_{k}\right)\right)
$$

where $\mathcal{F}$ is the SSO scheme (1) and $\mathcal{G}$ is the Gauss error function. In (4), we suppose that the $m$-dimensional stochastic random vector $\left\{\boldsymbol{\epsilon}\left(t_{k}\right)\right\}_{k \in\{1, \ldots, K\}}$ is an additive zero mean Gaussian error. The $m \times m$ covariance matrix of $\boldsymbol{\epsilon}\left(t_{k}\right)$ is denoted by $\mathbf{R}\left(t_{k}\right)$. As the sensitivity of $J$ varies with the atmospheric conditions $\mathbf{Z}\left(t_{k}\right)$, particularly with the surface wind speed, $\mathbf{R}\left(t_{k}\right)$ is assumed in principle to vary with time. 
The statistical parameters correspond to the vector and matrices that define the system (3)-(4). They are denoted by $\boldsymbol{\psi}$. We use the term "statistical parameters" of the state-space statistical model to make the difference with the 6 "physical parameters" $\boldsymbol{\theta}$ of the SSO scheme. Namely, the statistical parameters are the a priori probability density function (PDF) of the physical parameters, given by $\mathbf{x}^{b}$ and $\mathbf{B}$, and the covariance error matrices $\mathbf{Q}$ and $\mathbf{R}\left(t_{k}\right) \forall k \in\{1, \ldots, K\}$. We write $\boldsymbol{\psi}=\left(\mathbf{x}^{b}, \mathbf{B}, \mathbf{Q}, \mathbf{R}\right)$. The statistical parameters define the uncertainty of the state-space statistical model and play a central role on the quality and rate of convergence in the estimation of the physical parameters with the filtering and smoothing techniques described below.

The estimation of the statistical parameters $\psi$ is conducted maximizing the total likelihood function $\mathcal{L}$. This function is based on the PDF of the initial state $p\left(\mathbf{x}\left(t_{1}\right)\right)$, the conditional state evolution $p\left(\mathbf{x}\left(t_{k}\right) \mid \mathbf{x}\left(t_{k-1}\right)\right)$ and the observations conditionally to the state $p\left(\mathbf{y}\left(t_{k}\right) \mid \mathbf{x}\left(t_{k}\right)\right)$. The three PDFs are assumed to be normally distributed with the respective mean and covariances: $\mathbf{x}\left(t_{1}\right)-\mathbf{x}^{b}$ and $\mathbf{B}$, $\mathbf{x}\left(t_{k}\right)-\mathbf{x}\left(t_{k-1}\right)$ and $\mathbf{Q}, \mathbf{y}\left(t_{k}\right)-\mathcal{H}_{k}\left(\mathbf{x}\left(t_{k}\right)\right)$ and $\mathbf{R}\left(t_{k}\right)$. Finally, using the Markov property of the state-space model, the total likelihood function is the product of the PDF for all times $K$. It is given by

$$
\begin{aligned}
\mathcal{L}(\mathbf{x}, \boldsymbol{\psi})=p\left(\mathbf{x}\left(t_{1}\right)\right) \prod_{k=2}^{K} p & \left(\mathbf{x}\left(t_{k}\right) \mid \mathbf{x}\left(t_{k-1}\right)\right) \\
& \times \prod_{k=1}^{K} p\left(\mathbf{y}\left(t_{k}\right) \mid \mathbf{x}\left(t_{k}\right)\right) .
\end{aligned}
$$

In practice, this total likelihood function is approximated by its expectation conditionally to all the observations $\mathbf{y}_{1: K}=\mathbf{y}\left(t_{1}\right), \ldots, \mathbf{y}\left(t_{K}\right)$. This requires the computation of the state smoothed probabilities to be described below.

\section{Estimation technique}

The algorithm to estimate the physical and statistical parameters is described concisely here. A diagram with the main steps of the algorithm is shown in Figure 6. The algorithm starts with a proposed set of statistical parameters $\hat{\boldsymbol{\psi}}^{(1)}$ which do not need to be known precisely. Then, the statistical parameters are estimated maximizing the total likelihood function using the Expectation-Maximization algorithm. A loop is initiated which is composed by an expectation and a maximization step. The expectation step computes the expectations given in Appendix A via the EnKS. The maximization step consists basically in computing the optimal $\hat{\psi}^{(j)}$ from the known analytical expressions given in Appendix B. At each iteration $j$ of the EM algorithm, we compute the innovation likelihood $l$ given in Appendix C. It is commonly used to evaluate the quality of the state estimates and to compare state-space models with different statistical parameters ( for more details, see [Cappé et al.(2005)], p.140). If the innovation likelihood does not change significantly, the last estimated $\hat{\boldsymbol{\psi}}^{(j)}$ is returned. These optimal statistical parameters given by the Expectation-Maximization algorithm are finally used to initiate a last EnKF run which estimate the physical parameters.

\subsection{Expectation-Maximization algo- rithm}

The maximum likelihood estimates of the statistical parameters $\boldsymbol{\psi}$ are conducted using the EM algorithm proposed by [Dempster et al.(1977)]. This is a classical method used in the case of incomplete or missing data. This iterative algorithm is based on two steps: the expectation of the total log-likelihood function (E-step) and its maximization with respect to $\boldsymbol{\psi}$ (Mstep). The EM algorithm begins with an initial set of statistical parameters $\hat{\boldsymbol{\psi}}^{(1)}$. Then, repeating the E and M steps, the sequence of estimates $\hat{\boldsymbol{\psi}}^{(j)}$ yields increasing values of the expected log-likelihood and converges to the maximum likelihood estimates.

At the iteration $j$, the E-step consists in computing the expected total log-likelihood function conditionally to the total observations and the previous estimated statistical parameters. It is given by

$$
\mathcal{Q}\left(\boldsymbol{\psi} \mid \hat{\boldsymbol{\psi}}^{(j-1)}\right)=E\left[\log (\mathcal{L}(\mathbf{x}, \boldsymbol{\psi})) \mid \mathbf{y}_{1: K}, \hat{\boldsymbol{\psi}}^{(j-1)}\right] .
$$




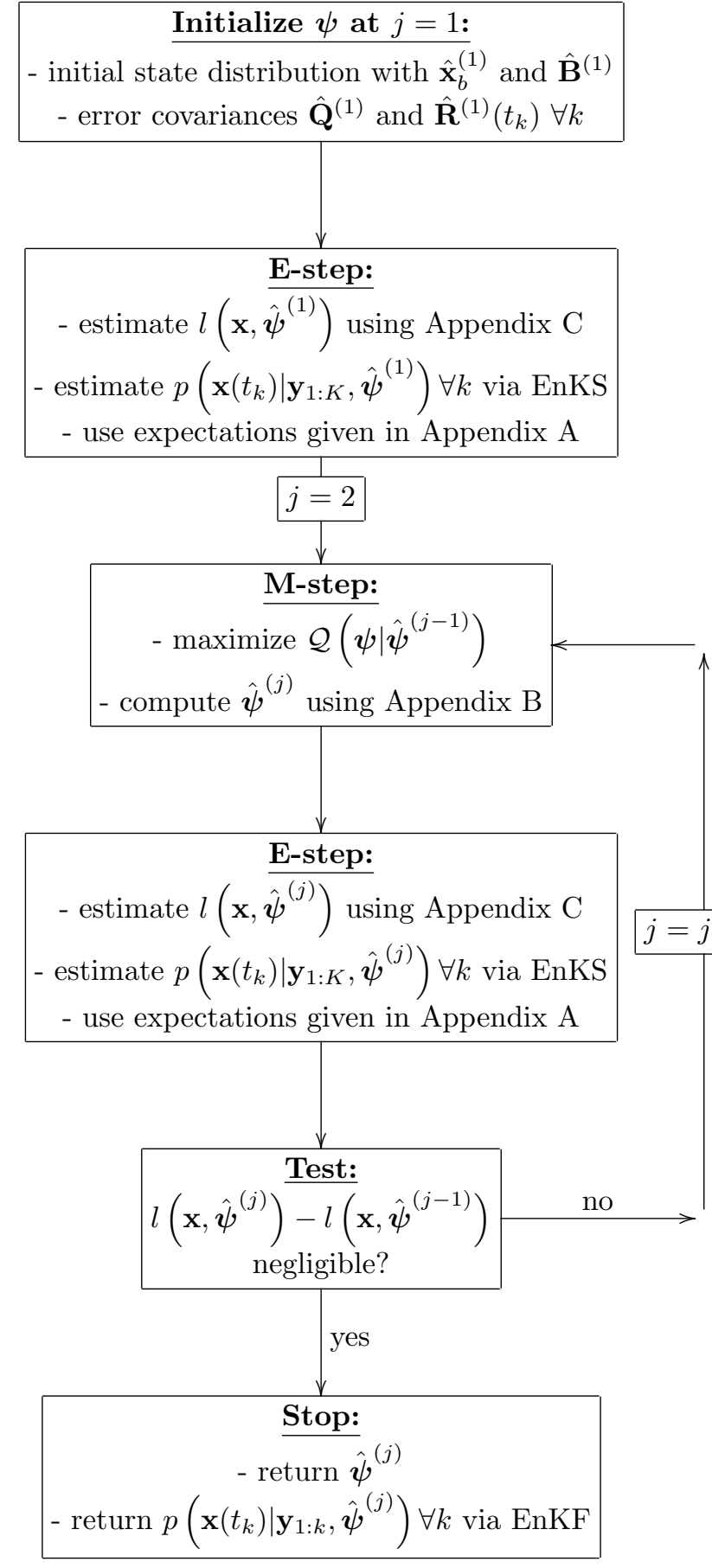

Figure 6: Diagram of the method based on the maximum likelihood estimates of the state-space model (3)-(4).
In the case of nonlinear state-space statistical models, the exact smoothed probabilities are not computable. Thus, we use the Monte Carlo approximations given by the EnKS. The conditional expectations given in Appendix A are then computed.

The M-step consists in maximizing $\mathcal{Q}\left(\boldsymbol{\psi} \mid \hat{\boldsymbol{\psi}}^{(j-1)}\right)$ with respect to $\psi$. We obtain a direct analytic form of the maximum likelihood estimates. The expressions are given in Appendix B. The derivations are not presented here (cf. [Tandeo et al.(2011)] for more details).

\subsection{Ensemble Kalman filter}

The EnKF algorithm used here is an adaptation of the one proposed by [Burgers et al.(1998)].

In the initial step of the EnKF algorithm, at time $t_{1}$, an ensemble of $\mathbf{x}$ 's composed by $N$ members is randomly generated. The members of the ensemble follow a Gaussian distribution given by the vector mean $\mathbf{x}^{b}$ and the covariance matrix B. The $N$ initial members are stored in the vectors $\mathbf{x}_{i}^{f}\left(t_{1}\right) \forall i \in$ $\{1, \ldots, N\}$.

In the update step, at each time $t_{k}$, we randomly $j=j+$ generate $N$ samples of $\boldsymbol{\eta}_{i}$ and $\boldsymbol{\epsilon}_{i} \forall i \in\{1, \ldots, N\}$ with respective covariances $\mathbf{Q}$ and $\mathbf{R}\left(t_{k}\right)$. Then, following (3), the $i$-member of the updated state is given by

$$
\mathbf{x}_{i}^{f}\left(t_{k}\right)=\mathbf{x}_{i}^{a}\left(t_{k-1}\right)+\boldsymbol{\eta}_{i}\left(t_{k}\right)
$$

and the mapping from the forecast state space to the observational space of the $i$-member is computed as

$$
\mathbf{y}_{i}^{f}\left(t_{k}\right)=\mathcal{H}_{k}\left(\mathbf{x}_{i}^{f}\left(t_{k}\right)\right) .
$$

The $N$ members of the ensemble are used to estimate the sample means of the propagated state in the state space and in the observational space denoted by $\mathbf{x}^{f}\left(t_{k}\right)$ and $\mathbf{y}^{f}\left(t_{k}\right)$ respectively.

In the analysis step, we follow [Pham(2001)] methodology which avoids the linearization of the observational operator. The Kalman gain is computed with

$$
\mathbf{K}\left(t_{k}\right)=\mathbf{P}_{x y}^{f}\left(t_{k}\right)\left(\mathbf{P}_{y y}^{f}\left(t_{k}\right)+\mathbf{R}\left(t_{k}\right)\right)^{-1},
$$


where $\mathbf{P}_{x y}^{f}\left(t_{k}\right)$ is the sample cross-covariance matrix and $\mathbf{P}_{y y}^{f}\left(t_{k}\right)$ is the sample covariance matrix, which are determined by

$$
\begin{aligned}
& \mathbf{P}_{x y}^{f}\left(t_{k}\right)=\frac{1}{N-1} \\
& \times \sum_{i=1}^{N}\left(\mathbf{x}_{i}^{f}\left(t_{k}\right)-\mathbf{x}^{f}\left(t_{k}\right)\right)\left(\mathbf{y}_{i}^{f}\left(t_{k}\right)-\mathbf{y}^{f}\left(t_{k}\right)\right)^{\top}
\end{aligned}
$$

and

$$
\begin{aligned}
& \mathbf{P}_{y y}^{f}\left(t_{k}\right)=\frac{1}{N-1} \\
& \times \sum_{i=1}^{N}\left(\mathbf{y}_{i}^{f}\left(t_{k}\right)-\mathbf{y}^{f}\left(t_{k}\right)\right)\left(\mathbf{y}_{i}^{f}\left(t_{k}\right)-\mathbf{y}^{f}\left(t_{k}\right)\right)^{\top} .
\end{aligned}
$$

In our case, the number of observations is larger than the dimension of the state space $(m>n)$ so that the matrix $\mathbf{P}_{y y}^{f}\left(t_{k}\right)+\mathbf{R}\left(t_{k}\right)$ is ill-conditioned making the matrix inversion difficult. Therefore, as described in [Evensen(2009)], Chapter 14, we compute the pseudo inverse of $\mathbf{P}_{y y}^{f}\left(t_{k}\right)+\mathbf{R}\left(t_{k}\right)$ taking into account $99 \%$ of the information given by the eigenvalues. Having $\mathbf{K}\left(t_{k}\right)$ from (10), the $N$ members of the ensemble are then updated by

$$
\mathbf{x}_{i}^{a}\left(t_{k}\right)=\mathbf{x}_{i}^{f}\left(t_{k}\right)+\mathbf{K}\left(t_{k}\right) \mathbf{d}_{i}\left(t_{k}\right)
$$

where the $m$-dimensional $\mathbf{d}_{i}\left(t_{k}\right) \forall i \in\{1, \ldots, N\}$ are the $\mathrm{N}$ innovation vectors in which we use perturbed observations such as $\mathbf{d}_{i}\left(t_{k}\right)=\mathbf{y}\left(t_{k}\right)+\boldsymbol{\epsilon}_{i}\left(t_{k}\right)-\mathbf{y}_{i}^{f}\left(t_{k}\right)$. Note that the sample covariance of the $\mathrm{N}$ innovations is $\mathbf{P}_{y y}^{f}\left(t_{k}\right)+\mathbf{R}\left(t_{k}\right)$. Finally, the updated analyzed state is represented by the sample mean $\mathbf{x}^{a}\left(t_{k}\right)$ and the sample covariance $\mathbf{P}^{a}\left(t_{k}\right)$.

\subsection{Ensemble Kalman smoother}

The backward recursions correspond to the EnKS algorithm proposed by [Evensen and Van Leeuwen(2000)]. It uses the results of the EnKF computed above.

In the initial step of the EnKS algorithm, at time $t_{K}$, we use the members of the filtered state, $\forall i \in$ $\{1, \ldots, N\}$, such as $\mathbf{x}_{i}^{s}\left(t_{K}\right)=\mathbf{x}_{i}^{a}\left(t_{K}\right)$ and $\mathbf{P}^{s}\left(t_{K}\right)=$ $\mathbf{P}^{a}\left(t_{K}\right)$.
Then, we proceed backward from $k=K-1$ to $k=1$. At each time $t_{k}$, we compute

$$
\mathbf{x}_{i}^{s}\left(t_{k}\right)=\mathbf{x}_{i}^{a}\left(t_{k}\right)+\mathbf{K}^{s}\left(t_{k}\right)\left(\mathbf{x}_{i}^{s}\left(t_{k+1}\right)-\mathbf{x}_{i}^{f}\left(t_{k+1}\right)\right)
$$

where $\mathbf{K}^{s}\left(t_{k}\right)$ is the $n \times n$ Kalman smoother gain matrix given by $\mathbf{P}^{a}\left(t_{k}\right)\left(\mathbf{P}^{f}\left(t_{k+1}\right)\right)^{-1}$. The Gaussian distribution of the updated state estimate is given by the sample mean and covariance respectively denoted by $\mathbf{x}^{s}\left(t_{k}\right)$ and $\mathbf{P}^{s}\left(t_{k}\right)$. The sample covariance of the state between two consecutive times is computed using

$$
\begin{aligned}
& \mathbf{P}^{s}\left(t_{k}, t_{k-1}\right)=\frac{1}{N-1} \\
& \times \sum_{i=1}^{N}\left(\mathbf{x}_{i}^{s}\left(t_{k}\right)-\mathbf{x}^{s}\left(t_{k}\right)\right)\left(\mathbf{x}_{i}^{s}\left(t_{k-1}\right)-\mathbf{x}^{s}\left(t_{k-1}\right)\right)^{\top} .
\end{aligned}
$$

\section{Results}

\subsection{Identical twin-experiment}

In order to evaluate the technique, twin experiments are used. In this case, the observations are obtained under the assumption of perfect model; in other words the SSO scheme is assumed to give the true tendencies when the physical parameters $\boldsymbol{\theta}^{t}=(1,1,0.25,1,1,0.5)$ are used as the true parameters. Then we suppose that the state, i.e. the physical parameters, is unknown and we try to estimate it via the state-space model (3)-(4) using the generated mountain drag observations. As schematized in Figure 6, we estimate the statistical parameters $\boldsymbol{\psi}=\left(\mathbf{x}^{b}, \mathbf{B}, \mathbf{Q}, \mathbf{R}\right)$ of the state-space model via the EM algorithm in order to improve the estimation of the physical parameters, $\boldsymbol{\theta}$. At iteration $j=1$, we deliberately initialize the state vector $\hat{\mathbf{x}}_{b}^{(1)}$ far from the true state values (corresponding to the true parameters). The corresponding covariance $\hat{\mathbf{B}}^{(1)}$ is chosen as the unit matrix $\mathbf{I}_{6}$ to generate large initial spreads of the members. Throughout the filter evolution, the members are randomly perturbed by the constant covariance matrix $\hat{\mathbf{Q}}^{(1)}=0.1 \times \mathbf{I}_{6}$ in (8). The covariance of the measurement errors in (9) is set to 
$\hat{\mathbf{R}}^{(1)}\left(t_{k}\right)=1000 \times \mathbf{I}_{100} \forall k \in\{1, \ldots, K\}$ that is of the same order as the mean value of the cost function $J$ given in (2). We use $N=100$ members and 25 iterations of the EM algorithm.

The innovation log-likelihood function and the total RMSE of the physical parameters for the conducted twin experiments are shown in Figure 7(a) as a function of the EM iteration. The results indicate that the innovation log-likelihood is a good synthetic indicator of the filter quality that follows the inverse variations of the total RMSE. In Figure 7(b), we decompose the total RMSE for each physical parameter. We find a good convergence of all the physical parameters after $j=10$ iterations except for $C_{d}, H_{N C}$ and $G$ that need more EM iterations. The evolution of these two last physical parameters as a function of time for different iterations $(j=1,10,25)$ of the EM algorithm are shown in Figure 8. For both physical parameters, the EM algorithm is able to adapt the filter conditions and to give, along the iterations $j$, more and more accurate initial distributions of the physical parameters (given by the $\mathbf{x}^{b}$ and $\mathbf{B}$ maximum likelihood estimates). However, at the last iteration $j=25$, the temporal convergence (near $k=20$ ) is higher than the other physical parameters (not shown here). Note that the results using deterministic values of $\boldsymbol{\psi}$ instead of estimating them via the maximum likelihood method, show the inability of the filter to converge to the solution $\boldsymbol{\theta}^{t}$. This is shown with the blue curves of Figure 8 corresponding to the first iteration of the EM algorithm (i.e. this could be interpreted as a standard EnKF estimation). Even if we use more realistic but uniform values of covariance matrices $\hat{\mathbf{B}}^{(1)}, \hat{\mathbf{Q}}^{(1)}$ and $\hat{\mathbf{R}}^{(1)}\left(t_{k}\right) \forall k \in\{1, \ldots, K\}$, the standard EnKF is unable to converge to a stable and accurate solution.

Figure 9 (a) shows the matrix $\mathbf{Q}$ after $25 \mathrm{EM}$ iterations. A negative correlation between $H_{N C}$ and $G$ physical parameters is clearly detected. This confirms the observation we made from Figure 5 in the weak sensitivity region of the cost function $J$. The elements of the $\mathbf{Q}$ estimated by the maximum likelihood method for the covariance between $H_{N C}$ and $G$ and the variances of $H_{N C}$ and $G$ are respectively $-1.5 \times 10^{-5}, 1.5 \times 10^{-5}$ and $3 \times 10^{-5}$. These variances correspond to the optimal perturbations of the members in (3) at each time of the filter. Note that the amplitude of $\mathbf{Q}$ tends to decrease along the iterations of the EM algorithm since the model becomes perfect and the observations are produced with the optimal physical parameters. Concerning the estimated amplitude of the observation error covariance $\mathbf{R}\left(t_{k}\right)$, i.e. the covariance of $\boldsymbol{\epsilon}\left(t_{k}\right) \forall k \in\{1, \ldots, K\}$, it varies with the forcing terms, particularly the surface wind speed conditions. The results for the low wind speed conditions are shown in Figure 9(b) and for the high wind speed conditions in Figure 9(c). We distinguish different parts on these estimated matrices. The top left and the bottom right parts correspond respectively to the zonal and meridional error covariances of the observation equation given in (4). The top right and bottom left parts correspond to the cross covariance between the zonal and meridional components. The $\mathrm{x}$-axis and $\mathrm{y}$-axis indicate the vertical level of the different components. For instance, the level $1000 \mathrm{hPa}$ is given in the indexes 1 and 51 whereas the level $5 \mathrm{hPa}$ is given in the indexes 50 and 100 . The results indicate a checkerboard structure in the covariances inside groups of vertical levels and especially a larger variability of the observation error in the levels close to the surface in both cases. We also remark a cross covariance between the zonal and meridional error terms in this altitude for the low wind speed conditions. The main difference between the two estimated matrices is the amplitude of the variability. In strong wind speed conditions, the variance is globally enhanced by a factor of $10^{3}$. Therefore, the method proposed here is able to model a flow dependent (typically the wind speed) and not necessarily diagonal error covariance matrix R. [Miyoshi et al.(2012)] has also proposed to retrieve the shape of $\mathbf{R}$ in a data assimilation problem conducting twin experiments. More precisely, they extended the adaptive estimation method proposed by [Li et al.(2009)] to include off-diagonal terms of $\mathbf{R}$.

We make two remarks on results that are not shown here. Firstly, the use of $N=500,1000$ members in the ensemble (not shown) gives similar results as the case with $N=100$ presented here. Thus, an ensemble of 100 members is sufficient to capture the highly nonlinear behavior of the SSO scheme and to estimate 
properly the statistical parameters of the state-space system. Secondly, the maximum likelihood statistical parameter $\boldsymbol{\psi}$ estimates are independent of the initial conditions of the EM algorithm. Different initial guess parameters $\hat{\mathbf{x}}_{b}^{(1)}$ and different covariances $\hat{\mathbf{B}}^{(1)}$ give similar rate of convergence.

\subsection{Changes in orography resolution}

When the resolution of a GCM is increased, or when a new dataset is used to feed the physical parameterizations, the physical parameters of the GCM need to be adjusted. There is no systematic way to produce these adjustments in the schemes so far. The technique introduced in this work can be used to do this. In particular, the standard parameters that are currently used in the SSO scheme shown in Table 1 have been manually tuned using PYREX data by [Lott and Miller(1997)]. This set of parameters are used operationally in the LMDz model. The tuning was conducted with a version of the SSO scheme that uses the low resolution orography $\left(10^{\prime} \times 10^{\prime}\right.$, see Figure 10(a)). Suppose that the higher resolution $\left(2^{\prime} \times 2^{\prime}\right.$, see Figure $10(\mathrm{~b})$ and $\left.[\mathrm{NOAA}(2001)]\right)$ orography dataset is used to improve LMDz at a given horizontal resolution. The parameters of the scheme should be adjusted for this new orography dataset. We conducted an experiment to examine if the technique is able to determine a new set of optimal parameters for this high resolution orography dataset. The conducted data assimilation experiments use the SSO tendencies predicted by the SSO scheme using the low resolution orography $\left(10^{\prime} \times 10^{\prime}\right)$ as observations. The assimilation then uses the SSO tendencies predicted with a higher resolution orography dataset. In this way, the operator $\mathcal{H}_{k}$ used in the assimilation has an error.

In this experiment in which the SSO scheme is not "perfect" due to the resolution change, we found that the results depend on the initial guess conditions, in opposition to the identical twin experiments. As convergence could not be reached easily, one hundred filter experiments with different random initial guess conditions $\hat{\mathbf{x}}_{b}^{(1)}$ of the EM algorithm were conducted. Among these 100 experiments, we find re-

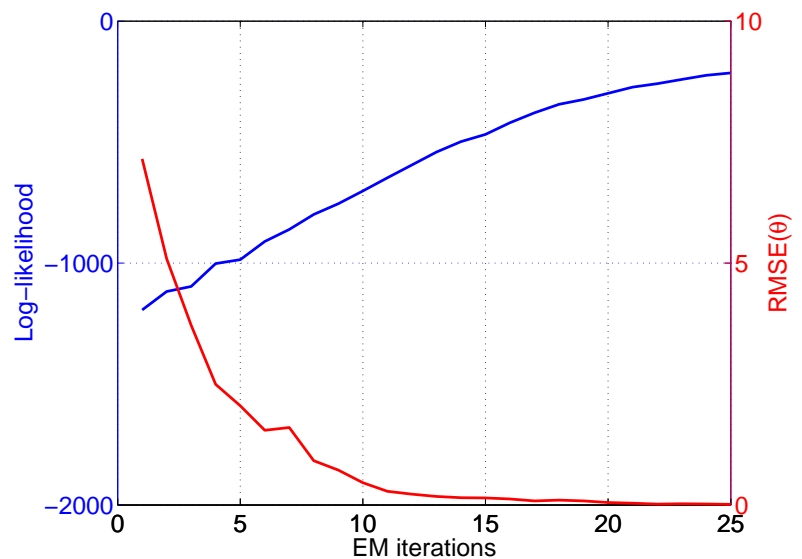

(a)

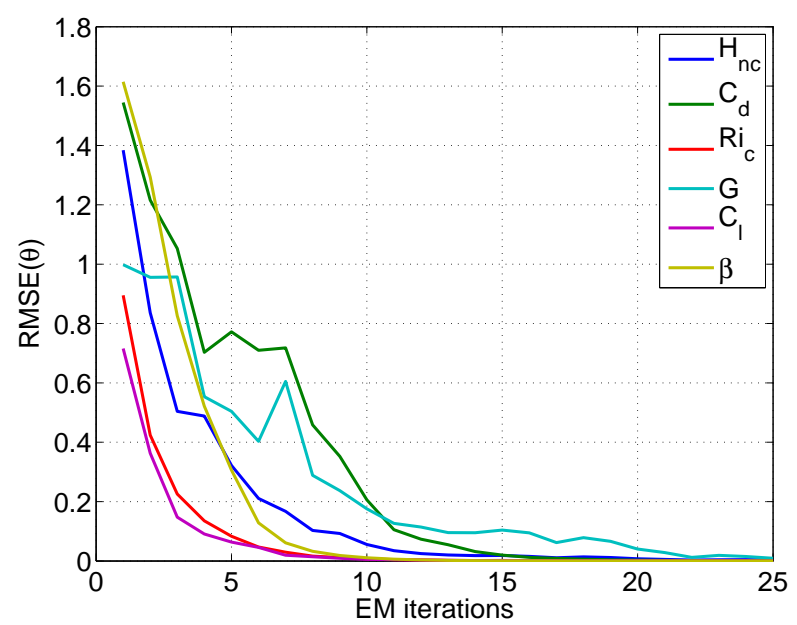

(b)

Figure 7: Evolution of (a) the innovation loglikelihood (left y-axis and blue line), the total physical parameter RMSE summed by time (right y-axis and red line) and (b) detailed RMSE of each physical parameters along $j=25$ iterations of the EM algorithm. 


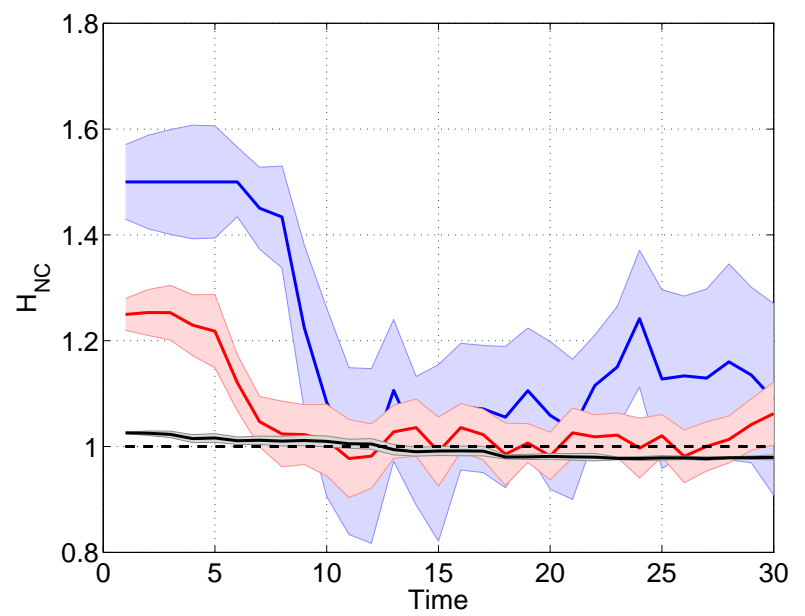

(a)

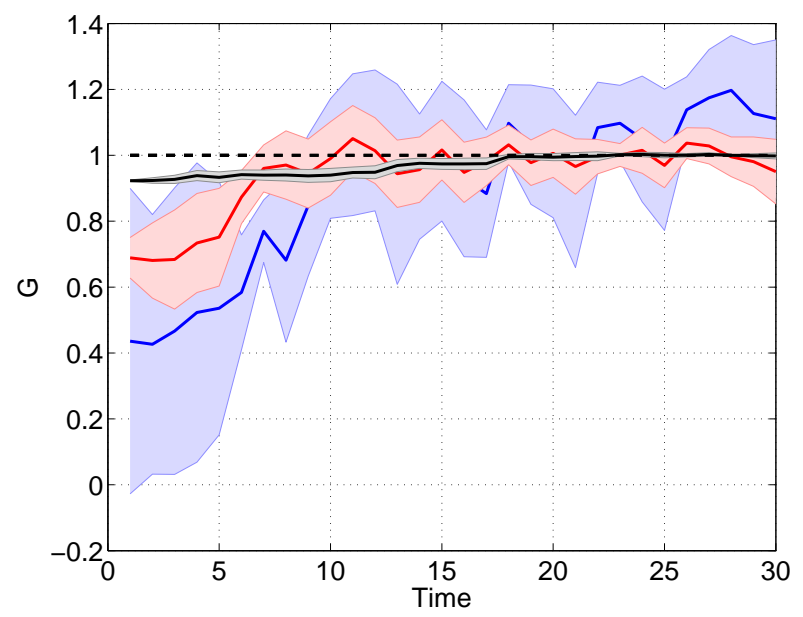

(b)

Figure 8: Evolution of the (a) $H_{N C}$ and (b) $G$ estimates with their $95 \%$ confidence interval along time (x-axis) for different iterations of the EM algorithm: $j=1$ (blue), $j=10$ (red) and $j=25$ (black). The straight lines correspond to the true physical parameter values. The physical parameters $(\boldsymbol{\theta}$, not $\mathbf{x})$ are shown.

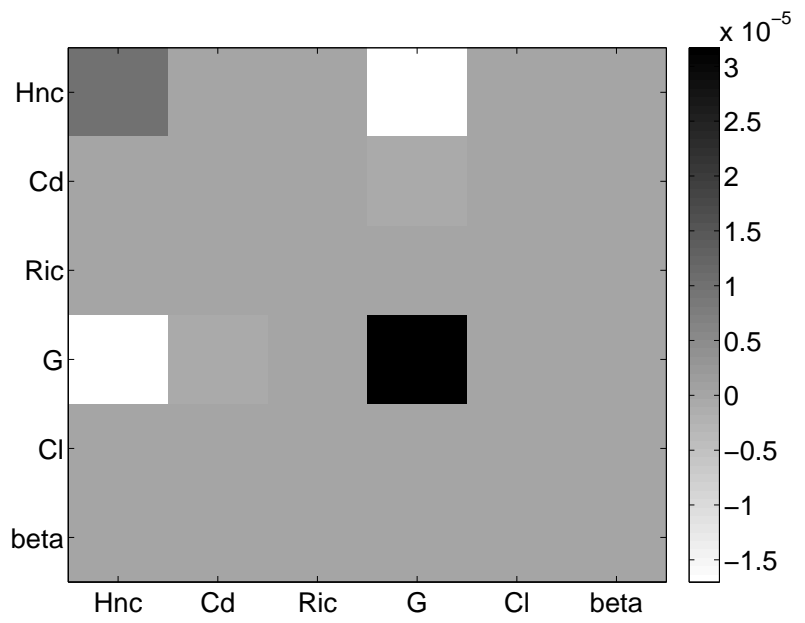

(a)

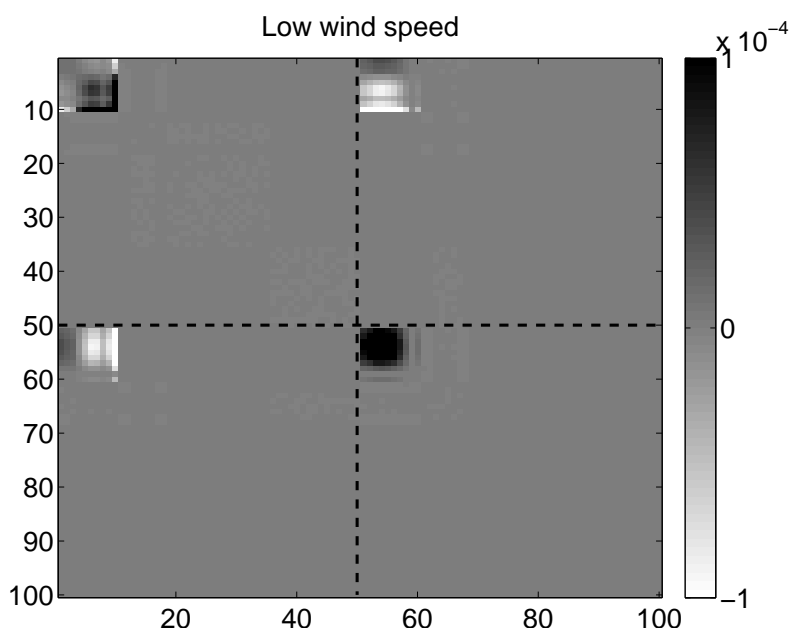

(b)

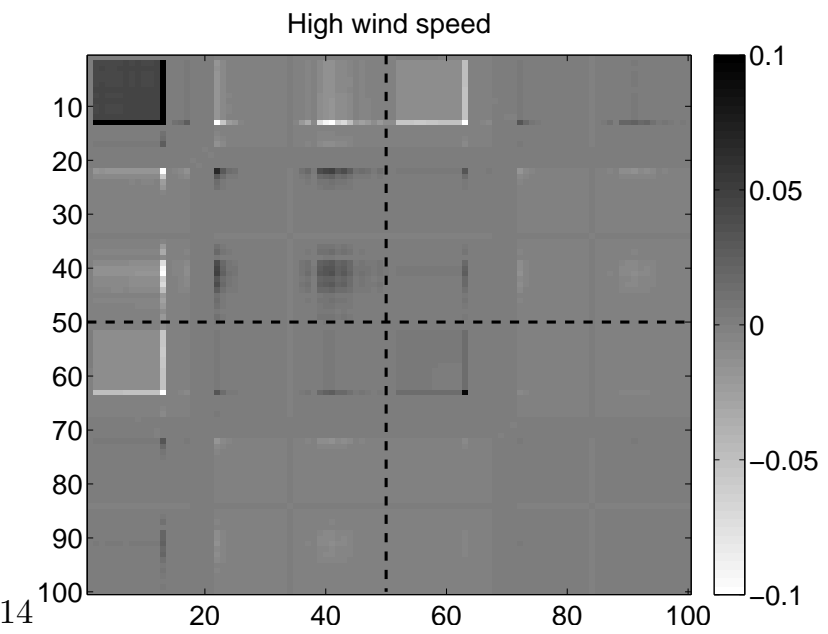

(c)

Figure 9: Maximum likelihood estimates after $j=25$ iterations of the EM algorithm of (a) $\hat{\mathbf{Q}}$, (b) $\hat{\mathbf{R}}\left(t_{5}\right)$ and (c) $\hat{\mathbf{R}}\left(t_{25}\right)$. The straight lines in (b) and (c) correspond to the limit between the zonal and meridional mountain drag error covariance of the observation equation. 
sults with recurrent estimations reaching to the same log-likelihood as those shown in Figure 11(a). The parameter estimations after $j=25$ EM iterations for five selected cases are shown in Figure 11(b).

From Figure 11(b), we notice that there is one parameter that does not need to be changed much when resolution is changed, $C_{l}$. This is not a surprise since $C_{l}$ is an almost linear lift coefficient, which is related to a mountain lift force which amplitude varies linearly with the difference between the mountain and valley height. We also find that $C_{d}$ needs to be reduced by a factor near two. Considering (16) in [Lott and Miller(1997)], the scheme measures the number of mountains that there is in a subgrid scale area, and multiply the low-level drag by this number of ridges. This yields a multiplicative factor in the mountain slope. When we move to a higher resolution grid, the estimate of the slope necessarily increases, so $C_{d}$ needs to decrease. The same conclusion could be drawn for the parameter $G$ that controls the gravity wave drag, but here the technique gives two possible solutions. One where $G$ is almost unchanged or a weak increase and one where it is decreased substantially, as expected. As the solution with unchanged $G$ is the most surprising, it is important to notice that they are also related to a smaller $\beta$, they therefore correspond to more trapped waves which apply low-level drag again. As at low level, it is $C_{d}$ that essentially controls the drag. We have therefore increased the gravity wave drag by increasing $G$ but placed that drag at low level where the effect is small compared to that of $C_{d}$. Another important result of the analysis is that the value of the critical Richardson number converges clearly to $R i_{c}=1.5$. As this high-resolution orography case likely has larger amplitude gravity waves, this larger Richardson number than the one used with the low-resolution orography dataset needs to be enhanced so that the waves propagate at high levels without breaking systematically at lower levels.

In general, the parameter estimations present a very large spread, except for $R i_{c}$, particularly for those parameters acting at low levels. For these, it should be remembered that the drag at low levels is always treated via implicit methods in part for stability, and in part because overestimated drags could yield wind reversals at low levels, which contradict the nature of drag forces. Clearly, the assimilation technique indicates us that some physical considerations should be done to make these parameters more efficient in controlling the drag. Among the possibilities, the SSO scheme does not consider that when there is several mountains in a gridbox area, some sheltering should be taken into account not to decelerate the same flow twice in a succession. This is currently handled implicitly by the scheme, but the low-level drag should take into account this horizontal sheltering when we increase the orography resolution.

Figure 11(b) shows that the filter converges towards two possible optimal states, in which $H_{N C}$ and $G$ clearly present bimodal distribution. This result is associated with the high correlation that was found in the cost function between $H_{N C}$ and $G$ as shown in Figure 5. The presence of model error in this imperfect model experiment appears to add complexity to the cost function with the presence of these two local minima. This is coherent with the results obtained by [Schirber et al.(2013)] in an online parameter estimation under the presence of model error. From a physical perspective, this bimodal result is not surprising since large $H_{N C}$ yields low blocking levels, and a more efficient mountain elevation to excite gravity waves. When there is a larger amount of gravity wave drag, a good fraction of the corresponding gravity waves is likely to break at low level, this may be an effect hidden in the low-level drag discussed before. Also, this bimodality may be inherent to the nonlinear low-level flow dynamics the scheme tries to represent.

Figure 12 shows the five profiles of the SSO tendency intensity (i.e. the norm of the SSO tendency) generated with the estimated parameters for two wind speed conditions. Figure 12(a) shows for weak surface wind conditions (on 5 July 2000) and Figure 12(b) for strong surface wind conditions (on 25 July 2000). In both surface wind conditions, the sets of estimated parameters with large $G$ tend to underestimate the low level drag (between $900 \mathrm{hPa}$ and $1000 \mathrm{hPa}$ ) and to overestimate the drag at high levels (between $650 \mathrm{hPa}$ and $900 \mathrm{hPa}$ ). On the other hand, the sets of estimated parameters with small $G$ and 


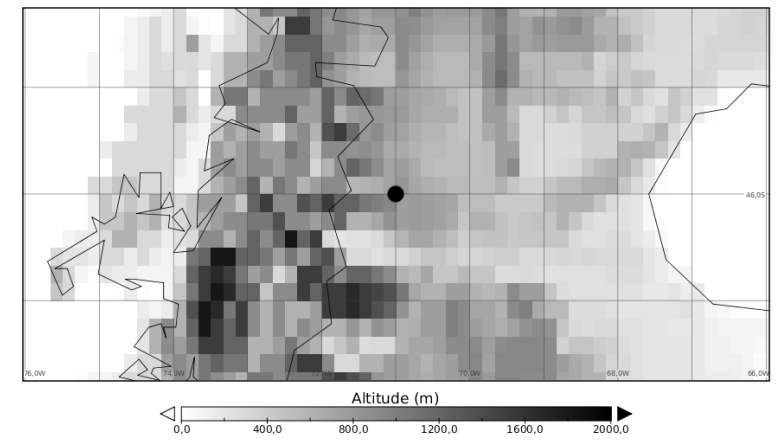

(a)

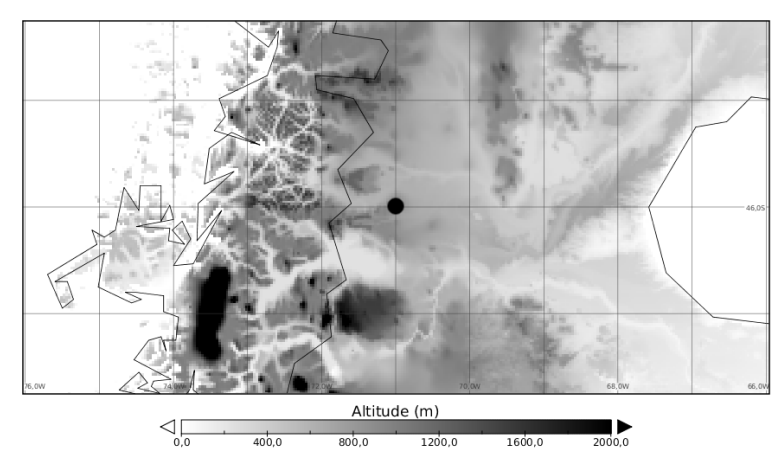

(b)

Figure 10: (a) Low $10^{\prime} \times 10^{\prime}$ and (b) high $2^{\prime} \times 2^{\prime}$ topographical resolution near the location $46^{\circ}$ south, $71^{\circ}$ west (black dot) in the South Andes.

(large $H_{n c}$ ) tend to overestimate the drag at low levels and also on higher levels (but they are relatively closer to the observed one than in the cases with large $G$ at those levels). The spread in $\beta$ parameter also appears to play a role.

\section{Conclusion and perspectives}

In this paper, we use a filtering technique to estimate the physical parameters of a subgrid-scale orographic scheme. The estimation is conducted offline, without estimating the state of the atmosphere and

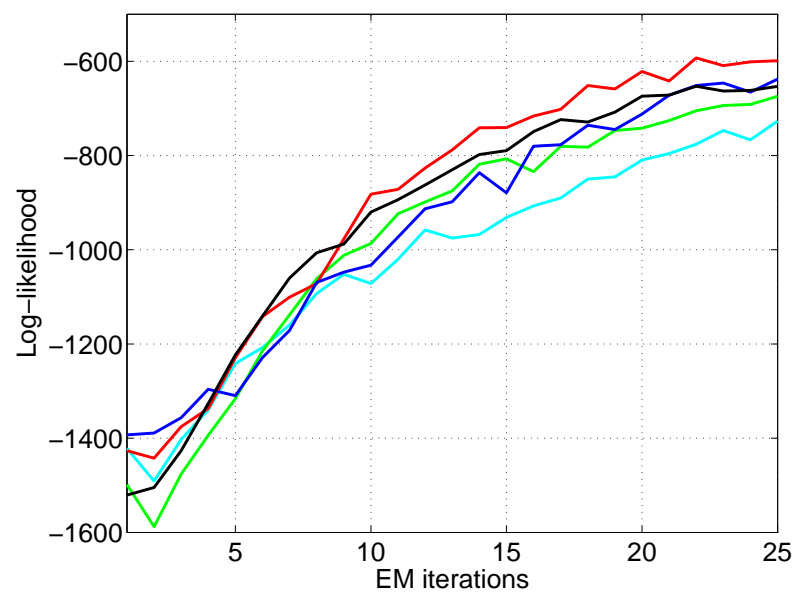

(a)
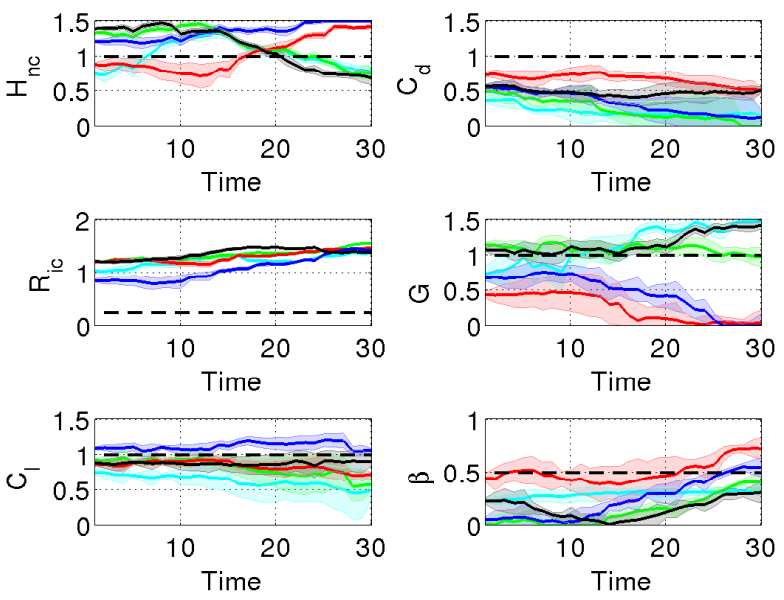

(b)

Figure 11: Evolution of 5 cases, with $N=100$ members and the high-resolution orographic scheme, of (a) the innovation log-likelihood along the EM iterations (x-axis) and (b) the physical parameters estimated by the EnKF along time (x-axis) at iteration $j=25$ of the EM algorithm. The straight dashed lines in (b) correspond to the physical parameter values of the low-resolution orographic scheme. In (b), the physical parameters $(\boldsymbol{\theta}$, not $\mathbf{x})$ are shown. 


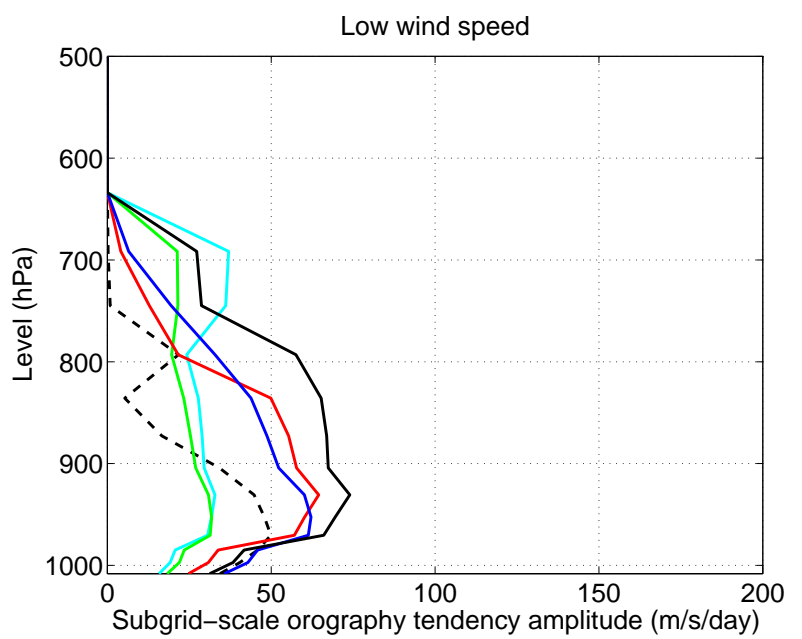

(a)

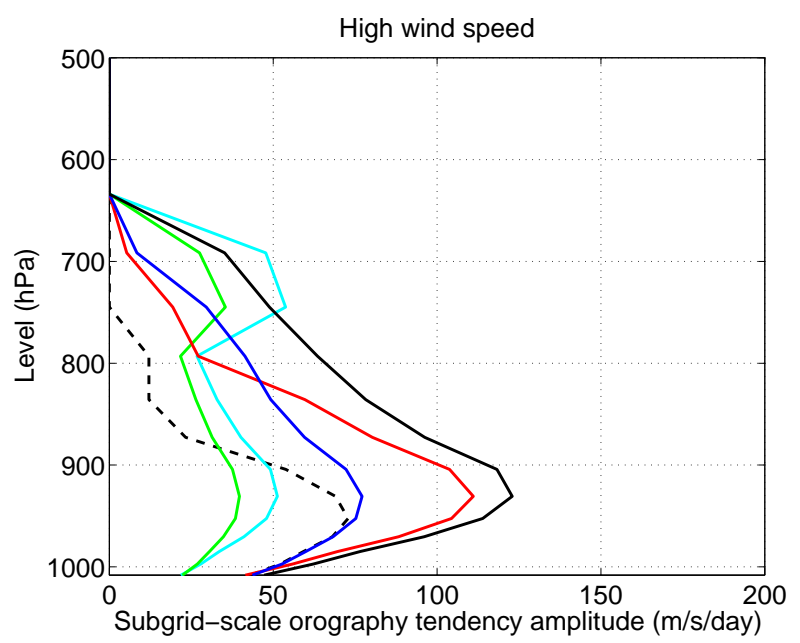

(b)

Figure 12: Profiles of the SSO tendency amplitude generated with the five sets of estimated parameters obtained with the SSO scheme using the high resolution dataset, (a) on 5 July 2000 and (b) on 25 July 2000. The profiles used as observations and generated with the SSO scheme using the low resolution dataset are shown with dashed lines. thus reducing the size of the state vector. As forcing terms, we use simulations of a general circulation model. The estimation problem is written as a nonlinear state-space system. This formulation is flexible and overcomes the main difficulties such as the boundaries on the physical parameters (strictly positive), the unknown background covariances and the high nonlinearity of the orographic scheme. In this state-space model, we suppose that the state and observation equations have additive Gaussian noise and that we know the a priori distribution of the physical parameters. The choice of these statistical parameters constitutes an important condition of convergence of the system to the true physical parameters. Thus, we estimate them via a maximum likelihood method. We use an iterative algorithm that computes the expected total log-likelihood function and maximizes it with respect to the statistical parameters.

The estimation technique is evaluated in a single vertical column and using synthetic observations (i.e., without using real observations but those produced by the SSO scheme). We imagine that an observational campaign takes place near the Perito Moreno Glacier in the Andes, where the topographical conditions are ideal to study mountain drag. First, we use twin experiments: we prescribe a true set of physical parameters and generate synthetic observations of mountain drag. Then, we apply the estimation technique using these generated observations and compare the estimated parameters to the true ones. The results indicate a convergence of the filter to the true parameters after $\sim 20$ iterations of the expectationmaximization algorithm. Even if the user initializes the error covariances and initial guess conditions with inappropriate values, these statistical parameters are iteratively updated and will converge towards the optimal values. The technique is able to detect correlations between parameters, to weight the observations as a function of the external forcing terms and to generate adaptive a priori information on the parameters. This overcomes the results obtained with deterministic values of statistical parameters which are usually arbitrarily prescribed since they are unknown.

We also examined if the estimation technique is 
useful to determine whether the physical parameter should be changed when the horizontal resolution of an input dataset of the general circulation model is increased. In this case, the SSO scheme is imperfect and our filter takes into account this model error adding Gaussian noises controlled by time dependent covariance matrices. The results show that our technique is a useful tool to determine the changes in the parameter when the resolution of the input orography dataset increases. However, model error degrades the estimated drag profiles, some features of the observed drag profile in Figure 12 can not be reproduced by the estimated drag profiles that use the high-resolution orography dataset. A technique with model bias treatment as in [Dee and Da Silva(1998)] may be required to diminish the differences in the drag profiles. We also detected that some parameters may have a range of values for which the RMSE and the likelihood (cf. Figure 11(a)) almost do not change. These results show that there are no sensitivity to these parameters and therefore determine a precise value of these parameters is not important. We attribute this to the fact that in the SSO scheme, a lot of drag is applied at low level and handled implicitly. In the scheme also, the low level drag is multiplied by the number of ridges present in the gridbox area, a number that is around 1 or 2 when the US Navy $10^{\prime} \times 10^{\prime}$ dataset becomes much larger when a more refined dataset is used. Ideally, we should take into account that when a mountain exerts a drag, a wake downstream is associated to it so that for the mountains in the lee but still in the gridbox, the incident flow should be much reduced. Currently, when we increase the orography resolution this effect is handled by an implicit treatment. Numerically this situation is satisfying, but clearly call for further understanding of the dynamical sheltering, and its impact on the large-scale flow. It may explain the difficulty to estimate the parameters and the difference between the drags generated with low and high resolutions orographic datasets in Figure 12.

The technique presented here is an efficient method to resolve offline physical parameter estimation. The advantages are (i) the flexibility of the state-space formulation that can be applied to a large number of applications, (ii) the ability to estimate the back- ground state and the eventually flow dependent and not necessarily diagonal error covariance matrices $\mathbf{Q}$ and $\mathbf{R}$ of the EnKF and (iii) the relative low computational cost of the technique where a relatively small number of members and few iterations of the expectation maximization algorithm are needed. One possible extension of the technique is the estimation of biases in more realistic cases with different kind of model errors.

This work is focused on the evaluation of the technique using first twin experiments and an experiment also with synthetic observations but using a higher resolution orographic dataset so that the model (used in the data assimilation system) in this case is imperfect. In a real application, the technique requires vertical profiles of small-scale momentum forcing. We envisage two possible sources of small-scale momentum forcing that can be used to constrain orographic parameters. The most significant one is from intensive observational campaigns that measure over mountainous areas. One of the most representative ones was the Pyrénée Experiment (PYREX, [Bougeault et al.(1990)]). Currently, there are several proposed campaigns to measure intensively over mountains, with airplanes, lidars and radiosondes. These combined instruments can give significant information on momentum fluxes and divergences of momentum fluxes. They are planned over the Andes, over New Zealand island and over Scandinavia. These potential campaigns could be an important source of observational data to estimate parameters of the subgrid-orography schemes using the proposed technique. A second possible observational data source of small-scale momentum forcing can be obtained from data assimilation techniques. [Pulido and Thuburn(2005)] show that fourdimensional variational assimilation can be used to estimate the missing momentum forcing term in the model equations. The technique is applied to obtain missing momentum forcing profiles in the middle atmosphere where a significant part of systematic model errors can be associated with gravity wave drag since the other physical parameterization active at those levels, the radiative transfer scheme, contains well-known parameters. On the other hand, in the troposphere several parameterizations are cou- 
pled between them so that the source of missing momentum is not readily identified with a particular parameterization. Therefore, the data assimilation techniques might be potentially useful to constrain subgrid orography schemes using only the momentum forcing profile in the stratosphere. However, the impact of model errors from different sources in the parameter estimation problem needs to be further investigated. Another point that needs to be further investigated in an actual application of this offline technique, is the possible feedbacks between the parameterization and the low-level flow, these feedbacks processes can affect the optimal parameters.

A follow-up work is to apply this estimation technique for online parameter estimation in strongly nonlinear systems. A first step will be to evaluate the method in a low dimension system. Parameter estimation in a low dimensional model was previously done in [Annan and Hargreaves(2004)] using deterministic values of the background state and the error covariance matrices. The advantage of applying our technique is to estimate them properly via the expectation maximization algorithm. Some first simulations we have performed give promising results. A simplified version of the method may also be useful in a larger dimension online parameter estimation problem, for instance when there are a few statistical parameters that are unknown and are essential to be estimated precisely.

\section{Acknowledgements}

This work was supported by ANPCyT Argentina under grant PICT 2007 No. 411., the ECOS-Sud project DIAGAC and by the LEFE project IAC. The authors would like to thank Dr. P. Ailliot and Dr. J.J. Ruiz for their helpful comments.

\section{Appendix A: E-step}

At each iteration $j$ of the EM algorithm, we need the following conditional expectations:

$$
\begin{aligned}
E\left[\mathbf{x}\left(t_{k}\right) \mathbf{x}\left(t_{k}\right)^{\top} \mid \mathbf{y}_{1: K}, \hat{\boldsymbol{\psi}}^{(j-1)}\right] & = \\
\mathbf{x}^{s}\left(t_{k}\right) \mathbf{x}^{s}\left(t_{k}\right)^{\top}+\mathbf{P}^{s}\left(t_{k}\right) & = \\
E\left[\mathbf{x}\left(t_{k}\right) \mathbf{x}\left(t_{k-1}\right)^{\top} \mid \mathbf{y}_{1: K}, \hat{\boldsymbol{\psi}}^{(j-1)}\right]= & = \\
\mathbf{x}^{s}\left(t_{k}\right) \mathbf{x}^{s}\left(t_{k-1}\right)^{\top}+\mathbf{P}^{s}\left(t_{k}, t_{k-1}\right) & = \\
E\left[\mathcal{H}_{k}\left(\mathbf{x}\left(t_{k}\right)\right) \mid \mathbf{y}_{1: K}, \hat{\boldsymbol{\psi}}^{(j-1)}\right]=\mathcal{H}_{k}\left(\mathbf{x}^{s}\left(t_{k}\right)\right) & = \\
E\left[\mathcal{H}_{k}\left(\mathbf{x}\left(t_{k}\right)\right) \mathcal{H}_{k}\left(\mathbf{x}\left(t_{k}\right)\right)^{\top} \mid \mathbf{y}_{1: K}, \hat{\boldsymbol{\psi}}^{(j-1)}\right]= & \\
\mathcal{H}_{k}\left(\mathbf{x}^{s}\left(t_{k}\right)\right) \mathcal{H}_{k}\left(\mathbf{x}^{s}\left(t_{k}\right)\right)^{\top}+\mathbf{P}_{y y}^{s}\left(t_{k}\right) &
\end{aligned}
$$

where $\mathbf{P}_{y y}^{s}\left(t_{k}\right)$ is the sample covariance of the $\mathcal{H}_{k}\left(\mathbf{x}_{i}^{s}\left(t_{k}\right)\right) \forall i \in\{1, \ldots, N\}$. 


\section{Appendix B: M-step}

The maximum likelihood estimates of the statistical parameters are given by:

$$
\begin{aligned}
\hat{\mathbf{x}}_{b}^{(j)=} & E\left[\mathbf{x}\left(t_{1}\right) \mid \mathbf{y}_{1: K}, \hat{\boldsymbol{\psi}}^{(j-1)}\right] \\
\hat{\mathbf{B}}^{(j)}= & \operatorname{Var}\left[\mathbf{x}\left(t_{1}\right) \mid \mathbf{y}_{1: K}, \hat{\boldsymbol{\psi}}^{(j-1)}\right] \\
\hat{\mathbf{Q}}^{(j)}= & \frac{1}{T-1} \sum_{k=2}^{T} E\left[\mathbf{x}\left(t_{k}\right) \mathbf{x}\left(t_{k}\right)^{\top} \mid \mathbf{y}_{1: K}, \hat{\boldsymbol{\psi}}^{(j-1)}\right] \\
& -\frac{1}{T-1} \sum_{k=2}^{T} E\left[\mathbf{x}\left(t_{k}\right) \mathbf{x}\left(t_{k-1}\right)^{\top} \mid \mathbf{y}_{1: K}, \hat{\boldsymbol{\psi}}^{(j-1) \mathrm{wi} \text { th th tiøn vect }}\right. \\
& -\frac{1}{T-1} \sum_{k=2}^{T} E\left[\mathbf{x}\left(t_{k}\right) \mathbf{x}\left(t_{k-1}\right)^{\top} \mid \mathbf{y}_{1: K}, \hat{\boldsymbol{\psi}}^{(j-1)}\right]^{\top} \\
& +\frac{1}{T-1} \sum_{k=2}^{T} E\left[\mathbf{x}\left(t_{k-1}\right) \mathbf{x}\left(t_{k-1}\right)^{\top} \mid \mathbf{y}_{1: K}, \hat{\boldsymbol{\psi}}^{(j-1)}\right] \\
\hat{\mathbf{R}}^{(j)}\left(t_{k}\right)= & \mathbf{y}\left(t_{k}\right) \mathbf{y}\left(t_{k}\right)^{\top} \\
& -E\left[\mathcal{H}_{k}\left(\mathbf{x}\left(t_{k}\right)\right) \mid \mathbf{y}_{1: K}, \hat{\boldsymbol{\psi}}^{(j-1)}\right] \mathbf{y}\left(t_{k}\right)^{\top} \\
& -\mathbf{y}\left(t_{k}\right) E\left[\mathcal{H}_{k}\left(\mathbf{x}\left(t_{k}\right)\right) \mid \mathbf{y}_{1: K}, \hat{\boldsymbol{\psi}}^{(j-1)}\right]^{\top} \\
& +E\left[\mathcal{H}_{k}\left(\mathbf{x}\left(t_{k}\right)\right) \mathcal{H}_{k}\left(\mathbf{x}\left(t_{k}\right)\right)^{\top} \mid \mathbf{y}_{1: K}, \hat{\boldsymbol{\psi}}^{(j-1)}\right]
\end{aligned}
$$

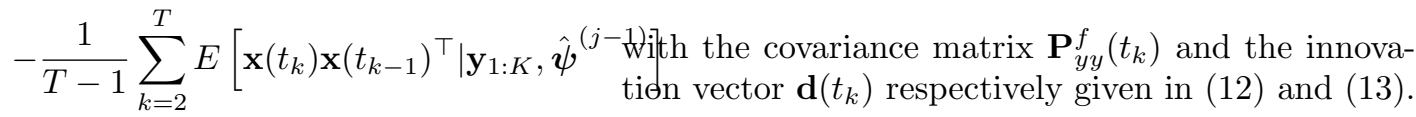

\section{Appendix C: innovation likeli-} hood

The innovation likelihood function is given by

$$
\begin{aligned}
& l(\mathbf{x}, \boldsymbol{\psi})= \\
& \prod_{k=1}^{K} \exp \left(-\frac{1}{2} \mathbf{d}\left(t_{k}\right)^{\top}\left(\mathbf{P}_{y y}^{f}\left(t_{k}\right)+\mathbf{R}\left(t_{k}\right)\right)^{-1} \mathbf{d}\left(t_{k}\right)\right) \\
& \quad \times(2 \pi)^{-p / 2}\left(\operatorname{det}\left(\mathbf{P}_{y y}^{f}\left(t_{k}\right)+\mathbf{R}\left(t_{k}\right)\right)\right)^{-1 / 2}
\end{aligned}
$$

tion vector $\mathbf{d}\left(t_{k}\right)$ respectively given in (12) and (13).

where the conditional expectations are computed in the E-step via the EnKS. 


\section{References}

[Anderson(2007)] Anderson JL. 2007. An adaptive covariance inflation error correction algorithm for ensemble filters. Tellus 59A: 210-224.

[Annan and Hargreaves(2004)] Annan JD, Hargreaves JC. 2004. Efficient parameter estimation for a highly chaotic system. Tellus 56A: $520-526$.

[Annan and Hargreaves(2007)] Annan JD, Hargreaves JC. 2007. Efficient estimation and ensemble generation in climate modelling. Philosophical Transactions of the Royal Society A: Mathematical, Physical and Engineering Sciences 365: 2077-2088.

[Bougeault et al.(1990)] Bougeault P, Jansa Clar A, Benech B, Carissimo B, Pelon J, Richard E. 1990. Momentum budget over the Pyrénées: The PYREX experiment. Bulletin of the American Meteorological Society 71: 806-818.

[Burgers et al.(1998)] Burgers G, Van Leeuwen PJ, Evensen G. 1998. Analysis scheme in the ensemble Kalman filter. Mon. Wea. Rev. 126: 1719 1724.

[Cappé et al.(2005)] Cappé O, Moulines E, Rydén T. 2005. Inference in hidden Markov models. Springer Science+Business Media: New York.

[Dee and Da Silva(1998)] Dee DP, Da Silva AM. 1998. Data assimilation in the presence of forecast bias. Q. J. R. Meteorol. Soc. 124: 269-295.

[Dempster et al.(1977)] Dempster AP, Laird NM, Rubin DB. 1977. Maximum likelihood from incomplete data via the EM algorithm. Journal of the Royal Statistical Society, Series B 39: 1-38.

[Evensen and Van Leeuwen(2000)] Evensen G, Van Leeuwen PJ. 2000. An ensemble Kalman smoother for nonlinear dynamics. Mon. Wea. Rev. 128: 1852-1867.

[Evensen(2009)] Evensen G. 2009. Data assimilation: the ensemble Kalman filter. Springer-Verlag: Berlin.
[Hertzog et al.(2012)] Hertzog A, Alexander MJ, Plougonven R. 2012. On the intermittency of gravity wave momentum flux in the stratosphere. J. Atmos. Sci. 69: 3433-3448.

[Hourdin et al.(2006)] Hourdin F, Musat I, Bony S and others. 2006. The LMDZ4 general circulation model: climate performance and sensitivity to parametrized physics with emphasis on tropical convection. Clim. Dyn. 27: 787-813.

[Hu et al.(2010)] Hu XM, Zhang F, NielsenGammon JW. 2010. Ensemble-based simultaneous state and parameter estimation for treatment of mesoscale model error: A real-data study Geoph. Res. Lett. 37: L08802.

[Ide et al.(1997)] Ide K, Courtier P, Ghil M, Lorenc AC. 1997. Unified notation for data assimilation: operational, sequential and variational. J. Meteorol. Soc. Japan 75: 181-189.

[Jackson et al.(2004)] Jackson C, Sen MK, Stoffa PL. 2004. An efficient stochastic Bayesian approach to optimal parameter and uncertainty estimation for climate model predictions. J. Climate 17: 2828-2841.

[Li et al.(2008)] Li F, Austin J, Wilson J. 2008. The strength of the Brewer-Dobson circulation in a changing climate: Coupled chemistry-climate model simulations. J. Climate 21: 40-57.

[Li et al.(2009)] Li H, Kalnay E, Miyoshi T. 2009. Simultaneous estimation of covariance inflation and observation errors within an ensemble Kalman filter. Q. J. R. Meteorol. Soc. 135: 523533.

[Liang et al.(2011)] Liang X, Zheng X, Zhang S, Wu G, Dai Y, Li Y. 2011. Maximum likelihood estimation of inflation factors on error covariance matrices for ensemble Kalman filter assimilation. Q. J. R. Meteorol. Soc. 138: 263-273.

[Lott(1995)] Lott F. 1995. Comparison between the orographic response of the ECMWF model and the PYREX 1990 data. Q. J. R. Meteorol. Soc. 121: $1323-1348$. 
[Lott and Miller(1997)] Lott F, Miller MJ. 1997. A new subgrid-scale orographic drag parameterization: Its formulation and testing. Q. J. R. Meteorol. Soc. 123: 101-127.

[Lott(1999)] Lott F. 1999. Alleviation of stationary biases in a GCM through a mountain drag parameterization scheme and a simple representation of mountain lift forces. Mon. Wea. Rev. 127: $788-801$.

[Lott et al.(2005)] Lott F, Fairhead L, Hourdin F, Levan P. 2005. The stratospheric version of LMDz: dynamical climatologies, arctic oscillation, and impact on the surface climate. Clim. Dyn. 25: 851-868.

[McLandress and Shepherd(2009)] McLandress C, Shepherd TG. 2009. Simulated anthropogenic changes in the Brewer-Dobson circulation, including its extension to high latitudes. $J$. Climate 22: 1513-1540.

[Miyoshi(2011)] Miyoshi T. 2011. The Gaussian approach to adaptive covariance inflation and its implementation with the local ensemble transform Kalman filter. Mon. Wea. Rev. 139: 1519 1535 .

[Miyoshi et al.(2012)] Miyoshi T, Kalnay E, Li H. 2012. Estimating and including observationerror correlations in data assimilation. Inverse Problems in Science and Engineering: published online.

[Palmer et al.(1986)] Palmer TN, Shutts GJ, Swinbank R. 1986. Alleviation of a systematic westerly bias in general circulation and numerical wea. prediction models through an orographic gravity wave drag parametrization. Q. J. R. Meteorol. Soc. 112: 1001-1039.

[Pham(2001)] Pham DT. 2001. Stochastic methods for sequential data assimilation in strongly nonlinear systems. Mon. Wea. Rev. 129: 1194-1207.

[Posselt and Bishop(2012)] Posselt DJ, Bishop CH. 2012. Nonlinear parameter estimation: Comparison of an ensemble Kalman smoother with a Markov chain Monte Carlo algorithm. Mon. Wea. Rev. 140: 1957-1974.

[Pulido and Thuburn(2005)] Pulido M, Thuburn J. 2005. Gravity wave drag estimation from global analyses using variational data assimilation principles. I: Theory and implementation. Q. J. R. Meteorol. Soc. 131: 1821-1840.

[Pulido and Thuburn(2008)] Pulido M, Thuburn J. 2008. The seasonal cycle of gravity wave drag in the middle atmosphere. J. Climate 21: 46644679 .

[Pulido et al.(2012)] Pulido M, Polavarapu S, Shepherd TG, Thuburn J. 2012. Estimation of optimal gravity wave parameters for climate models using data assimilation. Q. J. R. Meteorol. Soc. 138: 298-309.

[Ruiz et al.(2013)] Ruiz JJ, Pulido M, Miyoshi T. 2013. Estimating model parameters with ensemble-based data assimilation: A review. $J$. Meteorol. Soc. Japan 91: 79-99.

[Schirber et al.(2013)] Schirber S, Klocke D, Pincus R, Quaas J, Anderson JL. 2013. Parameter estimation using data assimilation in an atmospheric general circulation model: From a perfect toward the real world. Journal of Advances in Modeling Earth Systems 5: 58-70.

[Severijns and Hazeleger(2005)] Severijns CA, Hazeleger W. 2005. Optimizing parameters in an atmospheric general circulation model. $J$. Climate 18: 3527-3535.

[Sigmond et al.(2008)] Sigmond M, Scinocca JF, Kushner PJ. 2008. Impact of the stratosphere on tropospheric climate change. Geoph. Res. Lett. 35: L12706.

[Stainforth et al.(2005)] Stainforth DA, Aina T, Christensen C, Collins M, Faull N, Frame DJ, Kettleborough JA, Knight S, Martin A, Murphy JM and others. 2005. Uncertainty in predictions of the climate response to rising levels of greenhouse gases. Nature 433: 403-406. 
[Tandeo et al.(2011)] Tandeo P, Ailliot P, Autret E. 2011. Linear Gaussian state-space model with irregular sampling: application to sea surface temperature. Stoch. Environ. Res. Risk Assess. 25: 793-804.

[NOAA(2001)] U.S. Department of Commerce, National Oceanic and Atmospheric Administration, National Geophysical Data Center. 2001. 'NOAA ETOPO2 dataset, 2-minute Gridded Global Relief Data'. Available online at http://www.ngdc.noaa.gov/mgg/fliers/01mgg04.html.

[Wang and Bishop(2003)] Wang X, Bishop CH. 2003. A comparison of breeding and ensemble transform Kalman filter ensemble forecast schemes. J. Atmos. Sci. 60: 1140-1158.

[Wu et al.(2012)] Wu G, Zheng X, Wang L, Zhang S, Liang X, Li Y. 2012. A new structure for error covariance matrices and their adaptive estimation in EnKF assimilation. Q. J. R. Meteorol. Soc.: published online.

[Yang and Delsole(2009)] Yang X, Delsole T. 2009. Using the ensemble Kalman filter to estimate multiplicative model parameters. Tellus 61A: 601-609. 